\title{
A Super-Resolution Land-Cover Change Detection Method Using Remotely Sensed Images with Different Spatial Resolutions
}

\author{
Xiaodong Li, Feng Ling, Giles M. Foody, IEEE Fellow, and Yun Du
}

\begin{abstract}
X. Li, F. Ling and Y. Du are with the Key laboratory of Monitoring and Estimate for Environment and Disaster of Hubei province, Institute of Geodesy and Geophysics, Chinese Academy of Sciences, Wuhan 430077, PR China (e-mail: lingf@whigg.ac.cn). G. M. Foody is with School of Geography, University of Nottingham, University Park, Nottingham NG7 2RD, U. K.
\end{abstract}

Abstract-The development of remote sensing has enabled the acquisition of information on land-cover change at different spatial scales. However, a trade-off between spatial and temporal resolutions normally exists. Fine-spatial-resolution images have low temporal resolutions, whereas coarse spatial resolution images have high temporal repetition rates. A novel super-resolution change detection method (SRCD) is proposed to detect land-cover changes at both fine spatial and temporal resolutions with the use of a coarse-resolution image and a fine-resolution land-cover map acquired at different times. SRCD is an iterative method that involves endmember estimation, spectral unmixing, land-cover fraction change detection, and super-resolution land-cover mapping. Both the land-cover change/no-change map and from-to change map at fine spatial resolution can be generated by SRCD. In this study, SRCD was applied to synthetic multispectral image, Moderate-Resolution Imaging Spectroradiometer (MODIS) multispectral image and Landsat-8 Operational Land Imager (OLI) multispectral image. The land-cover from-to change maps are found to have the highest overall accuracy (higher than 85\%) in all the three experiments. Most of the changed land-cover patches, which were larger than the coarse-resolution pixel, were correctly detected.

Index Terms - Land-cover change detection, super-resolution mapping, the mixed pixel problem. 


\section{INTRODUCTION}

The detection of Earth's surface change serves as the basis for global change studies, and is critical to the understanding of the interactions between human and environmental systems. Remotely sensed data have become a primary data source for monitoring land use/cover distribution and its changes at different scales [1]. At the global scale, the coarse (low) spatial resolution images are the main data for land-cover monitoring. For instance, land-cover change products based on MODIS images at annual steps and $500 \mathrm{~m}$ spatial resolution for 2001-present have been used for global monitoring and assessment purposes. Coarse spatial resolution remote sensing systems have typically a high temporal repetition rate, which allow the timely detection of land-cover changes. For example, MODIS allows the entire surface of the earth to be monitored every 1 to 2 days since 2002. However, because of the relatively coarse spatial resolution of the images acquired the level of spatial detail detected is low. At the local-regional scale, land-cover change is often detected with the use of fine (high) spatial resolution remotely sensed images, such as those acquired by the Landsat Thematic Mapper (TM), Landsat Enhanced Thematic Mapper Plus (ETM+), and Systeme Pour l'Observation de la Terre (SPOT) sensors. However, owing to the trade-off between spatial and temporal resolution, fine-resolution images are often acquired at a relatively low temporal resolution. Thus regional land-cover change products are typically updated infrequently. For instance, the National Land Cover Database (NLCD) land-cover change product for the U.S.A. with a spatial resolution of $30 \mathrm{~m}$ is updated every 5 years approximately. Furthermore, fine-resolution images usually cover a relatively small area, making their use difficult for regional or global land-cover monitoring due to the great amount of processing time and labor required.

The accurate and timely detection of land-surface changes is a challenging task. Land-cover change detection is crucial to understand and quantify land-cover change, which is increasingly recognized as an important driver of global environmental change [2]. The most widely used land-cover change detection methods are classification-based (map-to-map) methods and spectrally based (image-to-image) 
methods, such as image differencing [3], vegetation index differencing [4], and change vector analysis [5]. However, these change detection methods are based on per-pixel comparison and require the same spatial resolution for bi-temporal images. Different resolution images are usually resampled during the spectral change detection process. A problem occurs when spectral modalities are different, and simple change detection (e.g., image differencing and thresholding) is often unreliable or impossible in case channels do not overlap well in the spectral ranges. Medium- or fine-resolution images are the main data sources for change detection; however, they are acquired at low temporal repetition rates, and land-cover changes are detected infrequently. By contrast, coarse-resolution images, which have high temporal repetition rates, are the main data sources of land-cover change detection at a global scale. Unfortunately, the aforementioned per-pixel-based change detection methods assume homogeneity within a single pixel, resulting in no quantifiable changes at the sub-pixel level. In fact, most coarse-resolution image pixels are composed of several land-cover/land-use types, and the mixed pixel problem seriously affects the change detection accuracy. Spectral unmixing or soft classification algorithms do not assign a mixed pixel to a single land-cover class but instead generate class area proportion or fraction images that represent proportional areas of different land-cover classes within mixed pixels. Spectral mixture analysis can be used to derive land-cover area proportion images, and changes can be detected by comparing the "before" and "after" area proportion images of each endmember[6]. Applying spectral unmixing to bi-temporal or a series of multitemporal images for change detection can potentially reveal important sub-pixel level information, such as the endmember abundance variation in a given location [7-10]. Spectral unmixing-based change detection methods provide the addition/subtraction of an endmember in the abundances and are based on the images with the same spatial resolution. In addition, He'garat-Mascle et al. [11] used images with different spatial resolutions to detect sub-pixel land-cover proportional change. A coarse-resolution image was unmixed and a fine-resolution image was spatially degraded to generate the bi-temporal class area proportion images. By comparing the bi-temporal coarse-resolution class area proportion images, the 
change in the class proportions of each coarse-resolution pixel was detected. However, only the coarse-resolution pixel change instead of the fine-resolution pixel change was detected, because spectral unmixing can only determine the coarse-resolution pixel land-cover area proportions and does not provide information on class labels at a sub-pixel scale.

Super-resolution land-cover mapping (SRM) is a technique used to generate land-cover maps with a finer spatial resolution than the input data. Various algorithms, such as pixel swapping algorithm [12, 13], Hopfield neural networks-based SRM [14], Markov-random-field-based SRM $[15,16]$, the spatial-spectral managed SRM [17, 18], spatial interpolation based SRM [19, 20], direct mapping based SRM [21], example-based SRM [22] and intelligence system based SRM [23], have been proposed to address the SRM problem. SRM predicts the spatial distribution of each class in each coarse-resolution pixel and provides more sub-pixel-scale land-cover information than spectral unmixing[24]. Traditionally, SRM is applied to a monotemporal coarse-resolution image to predict a fine-resolution land-cover map for the time period it represents. SRM is an ill-posed inverse problem because many fine-resolution land-cover maps can satisfy the SRM constraints, and traditional methods using a single image are limited in terms of the spatial detail represented and the accuracy of the final map produced. Additional datasets can, therefore, be adopted in SRM. A historic fine-resolution land-cover map may, for example, be used to enhance SRM. Ling et al. [25] first proposed a sub-pixel land-cover change mapping method by integrating a coarse-resolution image and a fine-resolution land-cover map that pre-dates the former. In this method, the sub-pixel class labels in the fine-resolution map with unchanged or increased class area proportions are preserved in the final fine-resolution land-cover map output from the SRM, and other sub-pixels are allocated based on the land-cover maximum spatial dependence model. Li et al. [26] proposed a spatial-temporal Markov-random-field-based SRM, in which the data on land-cover temporal dependence is the input to the analysis. Xu and Huang [27] proposed a spatial-temporal pixel-swapping algorithm based SRM, which extended the traditional pixel-swapping algorithm to involve sub-pixel temporal dependence. $\mathrm{Li}$ 
et al. [28] proposed a spatial-temporal Hopfield neural network based SRM, in which temporal transition information of sub-pixels was added compared with traditional Hopfield neural network based SRM.

Although the capability of SRM to extract fine-resolution land-cover information is increased with the use of a fine-resolution land-cover map that pre-dates the coarse-resolution image, the existing methods have three major limitations in the detection of land-cover change at fine spatial and temporal scales:

(1) With current SRM methods, the required information on endmembers, which can represent the spectral information of land-cover components, is typically assumed available. However, endmember information is often unavailable; thus, the extraction of endmembers in SRM is necessitated. Endmembers represent the spectrally pure components of a given image and are central to SRM. A considerable number of image endmember extraction algorithms, including the manual endmember selection tool [29], pixel purity index [30], N-FINDR [31], and automatic methods [32, 33], have been proposed in the recent years [34]. Manual endmember extraction methods are laborious, whereas iterative and automatic endmember extraction methods have been analyzed using only monotemporal imagery. When a fine-resolution land-cover map that pre-dates or post-dates the coarse-resolution image is available, useful information about land-cover configurations at a former or later time is available to facilitate SRM analysis.

(2) Current methods consider only the case wherein the fine-resolution land-cover map pre-dates the coarse-resolution image. Coarse-resolution remotely sensed images have been available for decades, particularly from such systems as MODIS and NOAA Advanced Very High Resolution Radiometer. With the development of remote sensing technology, remote-sensing systems with enhanced (finer) spatial resolution are increasing in number. As such, the fine-resolution images that post-date their corresponding coarse-resolution images may often be available in land-cover monitoring. However, this scenario has not been studied for current SRM methods.

(3) Although fine-resolution land-cover labels in each coarse-resolution pixel are 
predicted by SRM, land-cover change information is not provided. Map-to-map methods demonstrate a potential in fine-resolution change detection using images of different spatial resolutions, owing to the capability of SRM to generate land-cover map of a spatial resolution finer than that of the input image. Land-cover change information can be derived by comparing the land-cover maps of the same fine spatial resolution; these land-cover maps are generated from fine-resolution images based on hard classification and from coarse-resolution images based on SRM. This change detection method provides land-cover change information and trajectories that users typically desire, particularly a simple binary change/no-change map and the detailed from-to change trajectory information [6].

In order to address the above issues, a super-resolution change detection method (SRCD) is proposed. SRCD uses a combination of a fine-resolution land-cover map and a coarse-resolution image to detect land-cover change/no-change information and from-to information at fine spatial and temporal resolutions. The fine-resolution land-cover map can either pre-date or post-date the coarse-resolution image. The proposed method is based on the assumption that the land-cover class types are invariant during the period $[35,36]$. The endmembers are estimated and iteratively updated in SRCD. The remainder of this paper is organized as follows. Section II introduces the SRCD method. Section III examines the performance of SRCD using synthetic, MODIS, and Landsat images as well as for scenarios in which the fine-resolution land-cover map pre-dates and post-dates coarse-resolution image. Section IV concludes this paper.

\section{METHODS}


TABLE I.

LIST OF IMPORTANT VARIABLES AND METHOD DEFINITIONS

\begin{tabular}{|c|c|}
\hline $\begin{array}{l}\text { Variable and } \\
\text { method } \\
\text { abbreviations }\end{array}$ & Variable and method definitions \\
\hline SRCD & Super-resolution change detection method \\
\hline SRM & Super-resolution mapping \\
\hline $\mathbf{C}$ & Input coarse-resolution remote sensing image \\
\hline $\mathbf{F}$ & Input fine-resolution land-cover map \\
\hline $\mathbf{E}$ & Endmembers of all classes \\
\hline $\boldsymbol{e}_{n}$ & Endmembers of class $n$ \\
\hline$N$ & Number of classes in the coarse- and fine- resolutions images \\
\hline $\boldsymbol{c}_{i j}$ & Spectral signature vector of coarse-resolution pixel $(i, j)$ \\
\hline $\boldsymbol{p}_{i j}$ & $\begin{array}{l}\text { Class area proportion vector of all endmembers in coarse-resolution pixel } \\
(i, j)\end{array}$ \\
\hline$p_{i j n}$ & Class area proportion of the $n$-thendmember in coarse-resolution pixel $(i, j)$ \\
\hline$s$ & Scale factor between $\mathbf{C}$ and $\mathbf{F}$ \\
\hline$t$ & $\begin{array}{l}\text { Threshold value that determines whether a class in a coarse-resolution pixel } \\
\text { is changed }\end{array}$ \\
\hline$T$ & Acquisition time of the coarse-resolution image $\mathbf{C}$ \\
\hline $\mathbf{P}$ & Class area proportion image cube \\
\hline $\mathbf{P}_{(\mathrm{C})}$ & Class area proportion image cube generated from coarse-resolution image $\mathbf{C}$ \\
\hline $\mathbf{P}_{(\mathbf{F})}$ & Class area proportion image cube generated from fine-resolution map $\mathbf{F}$ \\
\hline$\Delta p_{i j n}$ & $\begin{array}{l}\text { Class area proportion difference value of class } n \text { in coarse-resolution pixel } \\
(i, j)\end{array}$ \\
\hline$\alpha_{i j, k}$ & The $k$-th fine-resolution pixel in coarse-resolution pixel $(i, j)$ \\
\hline$\lambda$ & Balance parameter in SRM \\
\hline $\mathbf{M}_{\text {class }}$ & Fine-resolution land-cover class (category) map \\
\hline $\mathbf{M}_{\mathrm{c} / \mathrm{n}}$ & Fine-resolution land-cover change/no-change map \\
\hline $\mathbf{M}_{\mathrm{f}-\mathrm{t}}$ & Fine-resolution land-cover from-to change map \\
\hline Intermediate $\mathbf{M}_{\mathrm{c} / \mathrm{n}}$ & $\begin{array}{l}\mathbf{M}_{\mathrm{c} / \mathrm{n}} \text { produced by the multiscale land-cover change strategy from } \mathbf{P}_{(\mathrm{C})} \text { and } \\
\mathbf{P}_{(\mathbf{F})}\end{array}$ \\
\hline Final $\mathbf{M}_{\mathrm{c} / \mathrm{n}}$ & $\mathbf{M}_{\mathrm{c} / \mathrm{n}}$ produced by per-pixel comparison between $\mathbf{M}_{\text {class }}$ and $\mathbf{F}$ \\
\hline
\end{tabular}

\section{A. SRCD Description}

Let $\mathbf{C}$ be the coarse-resolution image represented by an $I \times J \times B$ array, with $I$ and $J$ respectively denoting the width and height of the image, and $B$ denoting the number of bands. The $B \times 1$ vector representing the spectral signature of the coarse-resolution pixel $(i, j)$ is denoted by $\boldsymbol{c}_{i j}$. Let $\mathbf{F}$ be the input fine-resolution map 
with $I \times s \times J \times s$ pixels with $N$ land-cover classes in it. $\mathbf{F}$ can either pre-date or post-date $\mathbf{C}$. The scale factor between $\mathbf{F}$ and $\mathbf{C}$ is denoted by $s$, and each coarse-resolution pixel contains $s \times s$ fine-resolution pixels. Let $\mathbf{M}_{\text {class }}$ be the fine-resolution land-cover map with $I \times s \times J \times s$ pixels produced by SRM. Endmember matrix $\mathbf{E}$ is a $B \times N$ matrix, in which each column vector $\boldsymbol{e}_{n}$ corresponds to the spectrum vector of the $n$-th endmember. Let $\mathbf{P}$ be an $I \times J \times N$ array denoting the class area proportion cube. The $N \times 1$ vector representing the class area proportion of all endmembers in the pixel $(i, j)$ is denoted by $\boldsymbol{p}_{i j}$, and $p_{i j n}$ is the area proportion of the $n$-th endmember in the pixel $(i, j)$.

SRCD uses a coarse-resolution remotely sensed image $\mathbf{C}$ at acquisition time $T$ and a fine-resolution land-cover map $\mathbf{F}$ as inputs. SRCD outputs a fine-resolution land-cover change/no-change map $\mathbf{M}_{\mathrm{c} / \mathrm{n}}$ and a fine-resolution from-to change map $\mathbf{M}_{\mathrm{f}-\mathrm{t}}$. Both $\mathbf{M}_{\mathrm{c} / \mathrm{n}}$ and $\mathbf{M}_{\mathrm{f}-\mathrm{t}}$ are produced by comparing $\mathbf{F}$ with the estimated fine-resolution land-cover map $\mathbf{M}_{\text {class }}$ at time $T$.

Central to the proposed SRCD method is the estimation of $\mathbf{M}_{\text {class }}$ from $\mathbf{C} . \mathbf{M}_{\text {class }}$ and the endmember matrix $\mathbf{E}$ are the two interactive variables in SRCD. If $\mathbf{E}$ is known, $\mathbf{M}_{\text {class }}$ can be produced from $\mathbf{C}$ using SRM [25]. Frequently, $\mathbf{E}$ is an unknown variable and is estimated from $\mathbf{C}$, given the land-cover area proportion image cube $\mathbf{P}$ with the use of spectral unmixing [11, 37]. $\mathbf{P}$ can be derived by spatially degrading $\mathbf{M}_{\text {class, }}$, which is also an unknown variable in SRCD and is iteratively estimated and updated by determining $\mathbf{E}$ [11]. An iterative approach is used in SRCD to solve the endmember matrix $\mathbf{E}$ and fine-resolution land-cover map $\mathbf{M}_{\text {class }}$ coupling problem, that is, both variables $\mathbf{E}$ and $\mathbf{M}_{\text {class }}$ are estimated and updated iteratively.

The iterative SRCD starts from the estimation of the endmember matrix $\mathbf{E}$ at time $T$. At the first iteration, $\mathbf{F}$, which pre-dates or post-dates $\mathbf{C}$, is used as a substitute of $\mathbf{M}_{\text {class }}$ at time $T$, which would be accurate if all fine-resolution pixels are unchanged in the time period covered. At each iteration, the estimation of $\mathbf{E}$ is solved using a pseudo-inverse method, which employs $\mathbf{C}$ and $\mathbf{M}_{\text {class }}$ as inputs. Once $\mathbf{E}$ is estimated, $\mathbf{M}_{\text {class }}$ can be generated from $\mathbf{C}$ with the use of SRM. The estimated $\mathbf{M}_{\text {class }}$ is then used to re-estimate $\mathbf{E}$, and $\mathbf{M}_{\text {class }}$ is re-estimated with the iteratively updated $\mathbf{E} . \mathbf{E}$ and $\mathbf{M}_{\text {class }}$ 
are re-estimated and updated with each iteration. SRCD is iteratively run until convergence is achieved. Once SRCD converges, $\mathbf{M}_{\text {class }}$ is compared with the input $\mathbf{F}$ to produce the final land-cover change/no-change map $\mathbf{M}_{\mathrm{c} / \mathrm{n}}$ and from-to change map $\mathbf{M}_{\mathrm{f}-\mathrm{t}}$. The flowchart of SRCD is shown in Fig. 1.

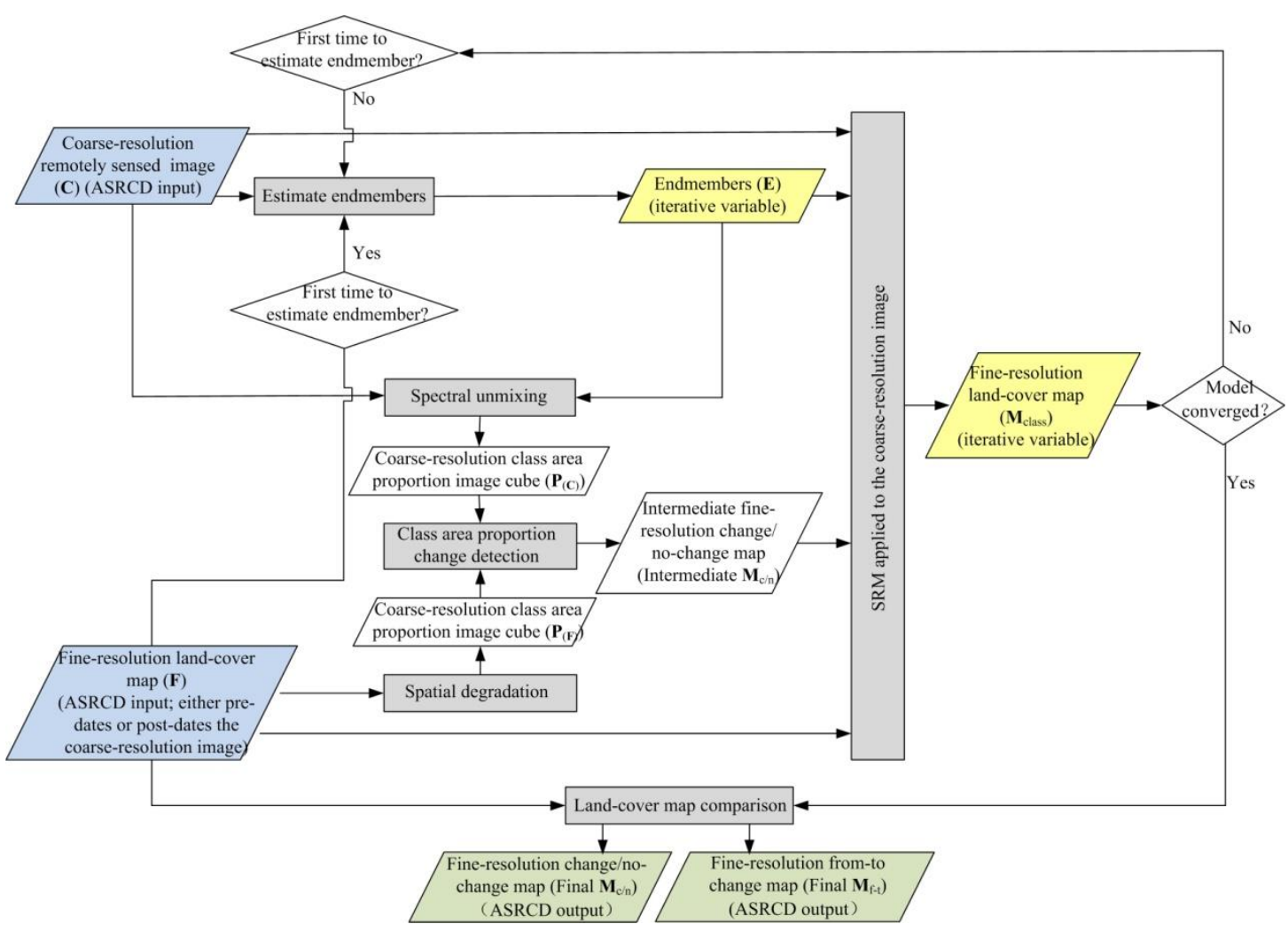

Fig. 1. The flowchart of the proposed SRCD method.

\section{B. Endmember Matrix E Estimation}

In SRCD, the endmember matrix $\mathbf{E}$ is estimated based on $\mathbf{C}$ and $\mathbf{M}_{\text {class }}$ at time $T$. $\mathbf{M}_{\text {class }}$ is degraded into the coarse-resolution land-cover area proportion image cube $\mathbf{P}$ by dividing the number of fine-resolution pixels of each class by the total number of fine-resolution pixels in a coarse-resolution pixel (i.e., $s^{2}$ ). The pixel spectrum is the result of a mixture of different endmember spectral signatures. We assume that the observed signals can be decomposed linearly (linear mixing assumption) and the measured contributions of the endmembers are proportional to their surface areas[38]. Based on the linear mixing assumption, the spectral signature $\boldsymbol{c}_{i j}$ for pixel $(i, j)$ can be represented by the following linear model: 


$$
\boldsymbol{c}_{i j}=\mathbf{E} \boldsymbol{p}_{i j}+\boldsymbol{\varepsilon}
$$

where $\varepsilon$ is an error term accounting for any noise in the imaging chain and other model inadequacies. In accordance with the mean square error minimization criterion, $\mathbf{E}$ in the image is estimated by searching for the solutions that minimize the squared error between the observed spectrum $\boldsymbol{c}_{i j}$ and the approximated spectrum, i.e.,

$$
[\tilde{\mathbf{E}}]=\operatorname{argmin}\left(\sum_{i=1}^{I} \sum_{j=1}^{J}\left[\boldsymbol{c}_{i j}-\sum_{n=1}^{N} p_{i j n} \boldsymbol{e}_{n}\right]^{2}\right) .
$$

Equation (2) is a pseudo-inverse problem. The endmember matrix $\mathbf{E}$ can be estimated according to the class area proportion image cube $\mathbf{P}$ and the coarse-resolution image $\mathbf{C}$ in (2).

\section{Fine-Resolution Land-Cover Map $M_{\text {class }}$ Estimation}

Once $\mathbf{E}$ is estimated, $\mathbf{M}_{\text {class }}$ at time $T$ can be derived using SRM applied to $\mathbf{C}$. In SRCD, SRM does not label all fine-resolution pixels in the image as traditional SRM methods do. Instead, it only updates the labels of changed fine-resolution pixels according to an intermediate fine-resolution change/no-change map (intermediate $\left.\mathbf{M}_{\mathrm{c} / \mathrm{n}}\right)$. The generation of $\mathbf{M}_{\text {class }}$ involves the production of the intermediate $\mathbf{M}_{\mathrm{c} / \mathrm{n}}$ and SRM. More specifically, the production of the intermediate $\mathbf{M}_{\mathrm{c} / \mathrm{n}}$ involves the generation of bi-temporal coarse-resolution images, the establishment of a multiscale land-cover change strategy to downscale these bi-temporal class area proportion image cubes to fine-resolution scale, and the determination of the class area proportion change thresholds in the bi-temporal class area proportion image cubes to quantify area proportion change in each coarse-resolution pixel. The procedures are explained below.

\section{1) Coarse-Resolution Land-Cover Class Area Proportion Estimation}

The coarse-resolution land-cover area proportion image cube $\mathbf{P}$ at time $T$ is estimated using spectral unmixing, given the coarse-resolution image $\mathbf{C}$ and endmember matrix E. In SRCD, the number of spectral bands is assumed to be no less than that of the endmembers ( $B \geq N$ ) to keep the effectiveness of the linear mixture 
model[39]. Based on the mean square error minimization criterion, the class area proportion vector $\boldsymbol{p}_{i j}$ in the coarse-resolution pixel $(i, j)$ is estimated by searching for the solutions that minimize the following expressions:

$$
\begin{gathered}
{\left[\tilde{\boldsymbol{p}}_{i j}\right]=\operatorname{argmin}\left[\boldsymbol{c}_{i j}-\sum_{n=1}^{N} p_{i j n} \boldsymbol{e}_{n}\right]^{2}} \\
0 \leq p_{i j n} \leq 1, \quad n=1, \cdots, N \\
\sum_{n=1}^{N} p_{i j n}=1 .
\end{gathered}
$$

Equation (3) is a constrained inverse problem, and two constraints in (4) and (5) are imposed on the objective function of (3) [40]. Then, $\mathbf{C}$ is unmixed and transformed into the class area proportion image cube $\mathbf{P}_{(\mathbf{C})}$ according to the linear mixture model.

$\mathbf{F}$ is spatially degraded into the class area proportion image cube $\mathbf{P}_{(\mathbf{F})}$. In the generation of $\mathbf{P}_{(\mathbf{F})}$, the class area proportion values of each class in each coarse-resolution pixel are calculated by dividing the number of fine-resolution pixels of each class by the total number of fine-resolution pixels in the coarse-resolution pixel (i.e., $s^{2}$ ).

2) Multiscale Land-Cover Change Strategy 


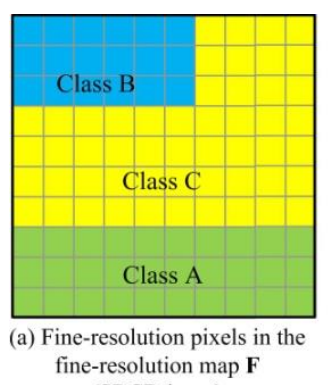

(SRCD input)

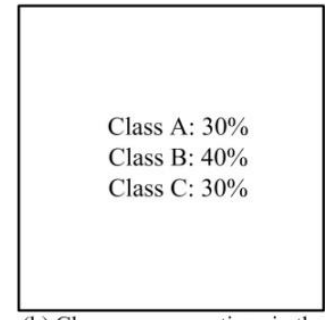

(b) Class area proportions in the coarse-resolution image pixel of $\mathbf{C}$ (SRCD input)

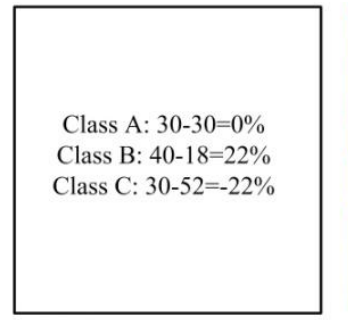

(c) Class area proportion changes from $\mathbf{F}$ to $\mathbf{C}$

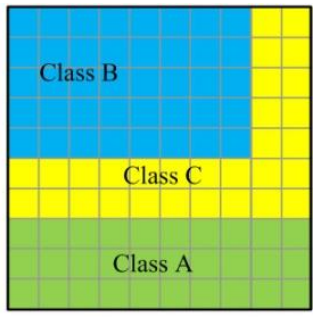

(d) Fine-resolution pixels that represent a time of $T$ (Reference map)

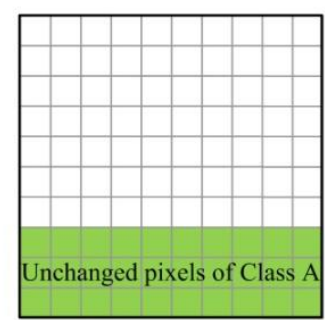

(e) Pixel locations of Class A accroding to SRCD

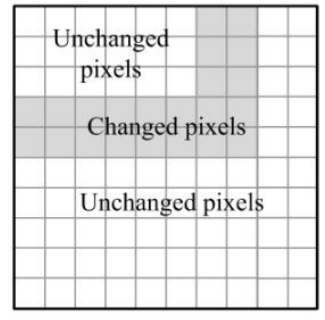

(h) Real change/no-change map

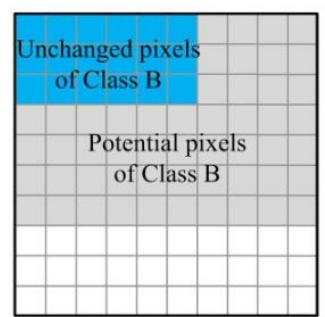

(f) Pixel locations of Class B accroding to SRCD

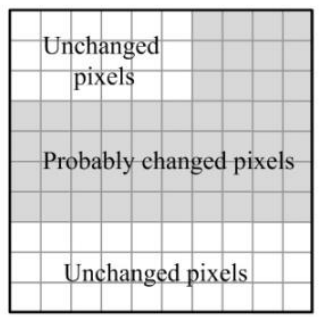

(i) SRCD intermediate change/no-change map $\mathbf{M}$

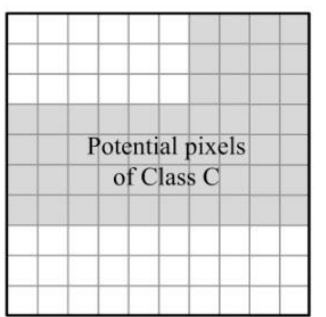

(g) Pixel locations of Class C accroding to SRCD

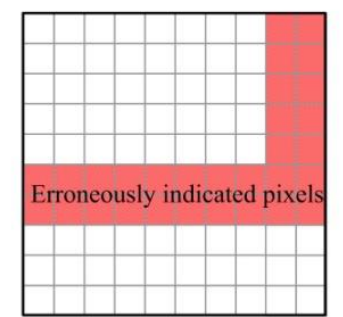

(j) Erroneously indicated pixels in the intermediate change/no-change map $\mathbf{M}_{c}$

Fig. 2. Production of the intermediate change/no-change map (intermediate $\mathbf{M}_{\mathrm{c} / \mathrm{n}}$ )

With bi-temporal coarse-resolution class area proportion image cubes $\mathbf{P}_{(\mathbf{C})}$ and $\mathbf{P}_{(\mathbf{F})}$, a multiscale land-cover change strategy is applied to downscale these class area proportion image cubes to produce the intermediate $\mathbf{M}_{\mathrm{c} / \mathrm{n}}$ by incorporating the input map F. The SRCD method is based on a simplistic view of land-cover change trajectory; this method compares the change in the class area proportion of each class from the fine-resolution map $\mathbf{F}$ to the coarse-resolution image $\mathbf{C}$. If the area proportion of a class in a coarse-resolution pixel appears to be unchanged (e.g., Class A in Fig. 2), then the number and location of the fine-resolution pixels of that class in $\mathbf{F}$ are unchanged in $\mathbf{C}$. If the area proportion of a class in a coarse-resolution pixel appears to have increased from $\mathbf{F}$ to $\mathbf{C}$ (e.g., Class $\mathbf{B}$ in Fig. 2), then the fine-resolution pixels of that class in $\mathbf{F}$ are unchanged, and some fine-resolution pixels in $\mathbf{F}$ from class(es) that decreased in class area proportion have transformed into that class in the coarse-resolution image pixel. If the area proportion of a class in a coarse-resolution pixel appears to have decreased from $\mathbf{F}$ to $\mathbf{C}$ (e.g., Class $\mathbf{C}$ in Fig. 2), 
then the fine-resolution pixels of that class in $\mathbf{F}$ include those that may have transformed into fine-resolution pixels of other classes, and no fine-resolution pixels in $\mathbf{F}$ are assumed to have transformed from other classes into that class in the coarse-resolution image pixel. Thus, the fine-resolution pixels of a class in $\mathbf{F}$ that decrease in area proportion probably contain the set of changed pixels. As a result, in F, the fine-resolution pixels with unchanged and increased class area proportions from $\mathbf{F}$ to $\mathbf{C}$ (e.g., Class A and Class B in Fig. 2) are probably the unchanged fine-resolution pixels, whereas the fine-resolution pixels with decreased class area proportions from F to $\mathbf{C}$ (e.g., Class $\mathbf{C}$ in Fig. 2) are probably the changed fine-resolution pixels in the coarse-resolution pixel. In addition, some unchanged fine-resolution pixels may be erroneously indicated as being changed pixels in the SRCD intermediate $\mathbf{M}_{\mathrm{c} / \mathrm{n}}$ (e.g., Class C in Fig. 2 (j)). Thus, SRCD may have overestimated the number of changed pixels but underestimated the number of unchanged pixels. As a result, the SRCD intermediate $\mathbf{M}_{\mathrm{c} / \mathrm{n}}$ contains commission errors of changed pixels and omission errors of unchanged pixels.

\section{3) Iterative Determination Method for Class Area Proportion Change Threshold}

In Fig. 2, the omission errors of unchanged pixels are permitted, whereas the commission errors of unchanged pixels are forbidden. This difference is because the SRM in SRCD only updates the fine-resolution pixel labels that are changed in the intermediate $\mathbf{M}_{\mathrm{c} / \mathrm{n}}$. If the commission error of unchanged pixels is large, the SRM in SRCD is essentially a traditional SRM that labels all fine-resolution pixels, and the information in $\mathbf{F}$ is merely used. The discrimination of unchanged fine-resolution pixels in the intermediate $\mathbf{M}_{\mathrm{c} / \mathrm{n}}$ is thus important and is implemented by injecting a threshold $t$ to quantify the amount of unchanged pixels in each coarse-resolution pixel. SRCD employs an iterative class area proportion change thresholding approach, which adopts the class area proportion image cube $\mathbf{P}_{(\mathbf{F})}$ based on $\mathbf{F}$ instead of $\mathbf{P}_{(\mathbf{C})}$ at the beginning because of the lack of endmember matrix $\mathbf{E}$ for unmixing $\mathbf{C}$. The class area proportion image cube $\mathbf{P}_{(\mathbf{F})}$ may be inaccurate in the initial iterations; thus, the determination of unchanged fine-resolution pixels should be precise to avoid high 
commission errors of unchanged pixels in the intermediate $\mathbf{M}_{\mathrm{c} / \mathrm{n}}$. Through the SRCD iterations, the endmember matrix $\mathbf{E}$ is re-estimated, and the accuracy of class area proportion image cube $\mathbf{P}$ increases. More potential unchanged fine-resolution pixels should be determined in the intermediate $\mathbf{M}_{\mathrm{c} / \mathrm{n}}$. The SRCD is iterated until the completion of a predefined number of iterations.

The iterative SRCD class area proportion change thresholding scheme is implemented. Let $\Delta p_{i j n}$ be the area proportion difference value of class $n$ in the coarse-resolution pixel $(i, j)$. It is calculated by subtracting the area proportion value of class $n$ in the pixel $(i, j)$ in the class area proportion image cube $\mathbf{P}_{(\mathbf{F})}$ from that in the class area proportion image cube $\mathbf{P}_{(\mathbf{C})}$. Let $t_{\text {real }}\left(t_{\text {real }}>0\right)$ be the real threshold value for detecting class area proportion change. On the basis of the multiscale land-cover change strategy, the fine-resolution pixels with unchanged class area proportion $\left(\Delta p_{i j n} \in\left[-t_{\text {real }}, t_{\text {real }}\right]\right)$ or increased class area proportion $\left(\Delta p_{i j n} \in\left(t_{\text {real }}, 1\right]\right)$ from $\mathbf{F}$ to $\mathbf{C}$ are labeled as unchanged in the intermediate $\mathbf{M}_{\mathrm{c} / \mathrm{n}}$. Let $t_{i n i}$ be the initial threshold value and $t$ be the iterative threshold value. The threshold value $t$ is iteratively decreased from a high value that approximates the higher bound of 1 to a low value that approximates the lower bound of $-t_{\text {real }}$ at an interval of $\Delta t \quad(\Delta t<0)$ to gradually detect the increased and unchanged pixels. Initially, $t$ is set to a high value, and fine-resolution pixels with progressively increasing class area proportion from $\mathbf{F}$ to $\mathbf{C}\left(\Delta p_{i j n}>t\right)$ are labeled as unchanged in the intermediate $\mathbf{M}_{\mathrm{c} / \mathrm{n}}$. Then, $t$ is gradually decreased at an interval of $\Delta t$, and more fine-resolution pixels with increased or unchanged class area proportion are labeled as unchanged based on the criterion $\Delta p_{i j n}>t$. When $t$ approximates the $-t_{\text {real }}$ value, the $\Delta p_{i j n}>t$ criterion ensures that all the fine-resolution pixels with increased class area proportion $\left(\Delta p_{i j n} \in\left(t_{\text {real }}, 1\right]\right)$ and unchanged class area proportion $\left(\Delta p_{i j n} \in\left[-t_{\text {real }}, t_{\text {real }}\right]\right)$ are correctly detected and labeled as unchanged in the intermediate $\mathbf{M}_{\mathrm{c} / \mathrm{n}}$. Through this procedure, the omission errors of the unchanged fine-resolution pixels and the commission errors of changed 
fine-resolution pixels are gradually reduced.

4) $S R M$

SRM in SRCD is used to produce the fine-resolution land-cover map $\mathbf{M}_{\text {class }}$ by updating fine-resolution pixels which are changed in the intermediate $\mathbf{M}_{\mathrm{c} / \mathrm{n}}$. In SRCD, the spatial-spectral managed algorithm proposed by Ling et. al [18] is modified only to label changed fine-resolution pixel labels. This SRM is simple implement and has fast convergence rate. In addition, this SRM has the similar objective function with the Markov-random-field-based SRM, yet it does not require the endmembers covariance matrix information as Markov-random-field-based SRM does [15].

The SRM algorithm comprises a spatial term, a spectral term, and a balance parameter. The spatial term encodes prior knowledge on land-cover spatial patterns assuming that spatially proximate observations of a given property are more similar than distant observations. The spectral term measures the spectral difference between the observed and synthetic pixel spectra. The balance parameter is utilized to balance the contributions of the spatial and spectral terms.

The objective function $(E)$ of SRM is characterized as

$$
E=\lambda \cdot E^{\text {spatial }}+(1-\lambda) E^{\text {spectral }}
$$

where $E^{\text {spatial }}$ is the spatial term, $E^{\text {spectral }}$ is the spectral term, and $\lambda$ is the balance parameter.

The SRM spatial term aims to maximize the spatial dependence of neighboring fine-resolution pixels based on the maximal spatial dependence model, given that the spatially proximate observations of a given property are more similar than more distant observations [41]. The spatial term for the $k$-th $\left(k=1, \cdots, s^{2}\right)$ fine-resolution pixel in coarse-resolution pixel $(i, j), a_{i j, k}$, is computed as

$$
E^{\text {spatial }}\left(c\left(a_{i j, k}\right)\right)=\sum_{l \in \mathrm{N}\left(a_{i j, k}\right)} \frac{1}{d\left(a_{i j, k}, a_{l}\right)} \cdot \delta\left(c\left(a_{i j, k}\right), c\left(a_{l}\right)\right) .
$$

$\mathrm{N}\left(a_{i j, k}\right)$ is a symmetric neighborhood that includes all fine-resolution pixels 
inside a square window whose center is $a_{i j, k}\left(a_{i j, k}\right.$ itself is not included in the window), and $a_{l}$ is a neighborhood fine-resolution pixel of $a_{i j, k}$ in $\mathrm{N}\left(a_{i j, k}\right)$. Here, the size of the neighborhood $\mathrm{N}\left(a_{i j, k}\right)$ was set to $2 \times s-1[16,42] . d\left(a_{i j, k}, a_{l}\right)$ is the Euclidian distance between $a_{i j, k}$ and $a_{l} \cdot c\left(a_{i j, k}\right)$ and $c\left(a_{l}\right)$ are the land-cover class labels for fine-resolution pixels $a_{i j, k}$ and $a_{l} \cdot \delta\left(c\left(a_{i j, k}\right), c\left(a_{l}\right)\right)$ equals to 0 if $c\left(a_{i j, k}\right)=c\left(a_{l}\right)$ and 1 otherwise.

The spectral term is utilized to link the observed remotely sensed image to the fine-resolution labeled map being modeled. The area proportion of class $n$ in pixel $(i, j), p_{i j n}$, is calculated by spatially degrading the fine-resolution label map $\mathbf{M}_{\text {class }}$ according to the scale factor. The SRM spectral term for coarse-resolution pixel $(i, j)$ is expressed as

$$
E^{\text {spectral }}(i, j)=\left(\boldsymbol{c}_{i j}-\sum_{n=1}^{N} p_{i j n} \boldsymbol{e}_{n}\right)^{\mathrm{T}}\left(\boldsymbol{c}_{i j}-\sum_{n=1}^{N} p_{i j n} \boldsymbol{e}_{n}\right) .
$$

Therefore, the objective function $(E)$ of SRM is calculated as

$$
E=\lambda \cdot \sum_{i=1}^{I} \sum_{j=1}^{J} \sum_{k=1}^{s^{2}} E^{\text {spatial }}\left(c\left(a_{i j, k}\right)\right)+(1-\lambda) \cdot \sum_{i=1}^{I} \sum_{j=1}^{J} E^{\text {spectral }}(i, j) .
$$

The accuracy of SRM is dependent on the balance parameter that balances the spatial and spectral terms. When the balance parameter yields a relatively low value for the spatial term, the land-cover class area proportions barely change, and the prior land-cover spatial patterns have little influence on $\mathbf{M}_{\text {class. }}$ Conversely, when the balance parameter yields a relatively high value for the spatial term, $\mathbf{M}_{\text {class }}$ may be oversmoothed, and the spectral information provided by the observed remotely sensed image may be lost [42].

An adaptive balance parameter estimation method for SRM in SRCD is proposed. The optimal balance parameter is estimated based on the energy balance analysis proposed by Tolpekin and Stein [42]. If a fine-resolution pixel label, with its true label $c\left(a_{i j, k}\right)=\alpha$, is assigned as a different label $\beta$ within a coarse pixel $(i, j)$, the change 
in the spatial term becomes $\Delta E_{\alpha \beta}^{\text {spatial }}$, and that in the spectral term becomes $\Delta E_{\alpha \beta}^{\text {spectral }}$ simultaneously. Therefore, to generate the correct class label on this fine-resolution pixel, the local contribution on the goal energy from the considered fine-resolution pixel should be lower for $c\left(a_{i j, k}\right)=\alpha$ than for $c\left(a_{i j, k}\right)=\beta$, and it necessitates the contribution of the spectral term to compensate for the gain of the spatial term. The limiting value of $\lambda$ is determined to balance the changes in the spatial and spectral terms in (10).

$$
\Delta E_{\alpha \beta}^{\text {spatial }} \leq \Delta E_{\alpha \beta}^{\text {spectral }}
$$

The change in the spatial term $\Delta E_{\alpha \beta}^{\text {spatial }}$ is formulated as

$$
\Delta E_{\alpha \beta}^{\text {spatial }}=q\left|\sum_{l \in \mathrm{N}\left(a_{i j, k}\right)} \phi\left(a_{l}\right)\left[\delta\left(\beta, c\left(a_{l}\right)\right)-\delta\left(\alpha, c\left(a_{l}\right)\right)\right]\right|=q \gamma
$$

where $\sum_{l \in \mathrm{N}\left(a_{i j, k}\right)} \phi\left(a_{l}\right)=1$ and $0 \leq q<\infty$ controls the overall magnitude of the weights of the spatial term. The parameter $\gamma$ depends on the neighborhood system size and the configuration of class labels $c\left(a_{l}\right)$ in the neighborhood $\mathrm{N}\left(a_{i j, k}\right)$. The parameter $\gamma$ value can be set as a constant $[16,42]$ or be estimated automatically [43]. In this paper, the parameter $\gamma$ is set to 0.03 according to [16, 42] for simplicity. The change in the spectral term $\Delta E_{\alpha \beta}^{\text {spectral }}$ before and after the update of the pair of fine-resolution pixel labels can be formulated as

$$
\Delta E_{\alpha \beta}^{\text {spectral }}=\left(\frac{\boldsymbol{e}_{\beta}-\boldsymbol{e}_{\alpha}}{s^{2}}\right)^{\mathrm{T}}\left(\frac{\boldsymbol{e}_{\beta}-\boldsymbol{e}_{\alpha}}{s^{2}}\right) .
$$

Once $\Delta E_{\alpha \beta}^{\text {spatial }}$ and $\Delta E_{\alpha \beta}^{\text {spectral }}$ are calculated, a range of the balance parameters that all satisfy (10) can be derived. Solving $\Delta E_{\alpha \beta}^{\text {spatial }}=\Delta E_{\alpha \beta}^{\text {spectral }}$ and recalling $\lambda=q /(1+q)$, the optimal value $\lambda^{*}$ is acquired as

$$
\lambda^{*}=\frac{1}{1+\frac{\gamma}{\Delta E_{\alpha \beta}^{\text {spectral }}}}
$$


If $\lambda>\lambda^{*}$, the model will result in oversmoothing. On the other hand, an extremely low value of $\lambda$ does not fully utilize the spatial information in the model. The value of $\Delta E_{\alpha \beta}^{\text {spectral }}$ is related to the class spectral separability, and the average value $\overline{\Delta E^{\text {spectral }}}$ is suggested to be used as a substitute for $\Delta E_{\alpha \beta}^{\text {spectral }}$ when class separability values for different pairs of classes are different [42].

$$
\overline{\Delta E^{\text {spectral }}}=\frac{\sum_{\alpha=1}^{N-1} \sum_{\beta=a+1}^{N} \Delta E_{\alpha \beta}^{\text {spectral }}}{\frac{N \cdot(N-1)}{2}} .
$$

Equation (13) is then rewritten as

$$
\lambda^{*}=\frac{1}{1+\frac{\gamma}{\Delta E^{\text {spectral }}}}
$$

\section{5) SRCD Energy Minimization}

The optimal $\mathbf{M}_{\text {class }}$ is generated by minimizing the SRCD objective function in (6). The SRM problem is characterized by a large solution space. Simulated annealing has been proposed to solve various global optimization problems, and avoid being trapped in the local minimum by controlling the acceptance of some inferior solutions which increase the objective function's value [44]. The acceptance of inferior solutions is dependent on a parameter $\kappa_{m} . \kappa_{m}$ is iteratively changed according to

$$
\kappa_{m}=\omega \times \kappa_{m-1} .
$$

The parameter $\omega \in(0,1)$ controls the rate of temperature decrease. With the decrement of $\kappa_{m}$, the inferior solutions will be accepted with low probability, and simulated annealing terminates when $\kappa_{m}$ is very small where the global minimum of the objective function is reached. The initial temperature $\kappa_{m}$ was set to 3 , the maximal iteration number was set to 120 , and the parameter $\omega$ was set to $0.9[16,42]$.

\section{The Proposed Algorithm}


SRCD is iterated until the threshold value reaches a predefined value or a predefined number of iteration has been completed. Once SRCD converged, $\mathbf{M}_{\text {class }}$ is compared with $\mathbf{F}$ to generate the fine-resolution land-cover change/no-change map $\mathbf{M}_{\mathrm{c} / \mathrm{n}}$ and from-to change map $\mathbf{M}_{\mathrm{f}-\mathrm{t}}$ through per-pixel comparison. The false code of SRCD is shown below: 
Objective: Estimate fine-resolution change/no-change map $\mathbf{M}_{\mathrm{c} / \mathrm{n}}$ and from-to change map $\mathbf{M}_{\mathrm{f}-\mathrm{t}}$

Input: Fine-resolution land-cover map $\mathbf{F}$, coarse-resolution image $\mathbf{C}$, scale factor $s$ between $\mathbf{F}$ and $\mathbf{C}$.

1. Initialization:

Set the initial threshold $t_{i n i}$, step threshold $\Delta t$, the initial iteration number ite $=0$ and total iteration number ite $_{\text {total }}$

2. Using $\mathbf{F}$ as the initial $\mathbf{M}_{\text {class }}$

3. Iteratively SRCD

do

\{

ite $=$ ite +1

Spatially degrade $\mathbf{M}_{\text {class }}$ to class area proportion image cube $\mathbf{P}_{(\mathbf{M})}$ (class area proportion image cube calculated from $\mathbf{M}_{\text {class }}$ ) according to scale factor $s$

Estimate endmember matrix $\mathbf{E}$ using $\mathbf{C}$ and $\mathbf{P}_{(\mathbf{M})}$ based on linear mixture model

Unmix $\mathbf{C}$ based on $\mathbf{E}$ to $\mathbf{P}_{(\mathbf{C})}$ (class area proportion image cube calculated from $\mathbf{C}$ )

Spatially degrade $\mathbf{F}$ to class area proportion image cube $\mathbf{P}_{(\mathbf{F})}$ (class area proportion image cube calculated from $\mathbf{F}$ ) according to scale factor $s$

Calculate class area proportion difference image $\mathbf{P}_{(\mathrm{C})^{-}} \mathbf{P}_{(\mathbf{F})}$

Update threshold $t$ value: $t=t_{\text {ini }}+$ ite $\times \Delta t$

Detect the area proportion change of each class in each coarse-resolution pixel

Generate the intermediate $\mathbf{M}_{\mathrm{c} / \mathrm{n}}$ based on threshold $t$

Use SRM to update labels of fine-resolution pixels in $\mathbf{M}_{\text {class }}$ which are changed in the intermediate $\mathbf{M}_{\mathrm{c} / \mathrm{n}}$

\}

until ite $=$ ite $_{\text {total }}$

4. Producing the final $\mathbf{M}_{\mathrm{c} / \mathrm{n}}$ and $\mathbf{M}_{\mathrm{f}-\mathrm{t}}$

Per-pixel comparison of $\mathbf{F}$ and $\mathbf{M}_{\text {class }}$

Result: $\mathbf{M}_{\mathrm{c} / \mathrm{n}}$ and $\mathbf{M}_{\mathrm{f}-\mathrm{t}}$

\section{E. Accuracy Metric}

The pixel-based quality metrics were used to assess the accuracy of $\mathbf{M}_{\mathrm{c} / \mathrm{n}}$ and $\mathbf{M}_{\mathrm{f}-\mathrm{t}}$ by comparing the result change maps with the reference data. The accuracies of the entire image $\mathbf{M}_{\mathrm{c} / \mathrm{n}}$ and $\mathbf{M}_{\mathrm{f}-\mathrm{t}}$ were assessed using the overall accuracy value [45]. The per-class accuracies of the changed and unchanged pixels in $\mathbf{M}_{\mathrm{c} / \mathrm{n}}$ and the per-class 
accuracies of the different land-cover from-to changes in $\mathbf{M}_{\mathrm{f}-\mathrm{t}}$ were also assessed. The accuracies of the changed and unchanged pixels in the intermediate $\mathbf{M}_{\mathrm{c} / \mathrm{n}}$ produced by the multiscale land-cover change strategy from $\mathbf{P}_{(\mathrm{C})}$ and $\mathbf{P}_{(\mathbf{F})}$ or final $\mathbf{M}_{\mathrm{c} / \mathrm{n}}$ produced by per-pixel comparison between $\mathbf{M}_{\text {class }}$ and $\mathbf{F}$ were measured using the omission error, commission error, and $\mathrm{F}_{1}$-score. The $\mathrm{F}_{1}$-score is the harmonic mean of precision, which represents the measure of exactness or quality, and recall, which represents the measure of completeness or quantity [46]. The per-class accuracies of different land-cover from-to changes in $\mathbf{M}_{\mathrm{f}-\mathrm{t}}$ were assessed using the producer's and user's accuracies.

\section{EXPERIMENTS AND RESULTS}

Experiments using synthetic multispectral image, real MODIS multispectral remotely sensed image and real Landsat- 8 multispectral image were conducted in order to assess the proposed SRCD method. The fine-resolution map post-dated the coarse-resolution image in the synthetic image experiment, and pre-dated the coarse-resolution image in the MODIS and Landsat- 8 image experiments. SRCD is not compared with other methods, because there is, to our knowledge, no other method focusing on the extraction of fine-resolution land-cover change information using different spatial resolution images without prior endmember information.

\section{A. Synthetic Image Experiment}

\section{1) Data Preparation}

A synthetic multispectral image was used in this experiment. The test data were obtained based on NLCD in 2001 and 2006, respectively. NLCD is a land-cover classification scheme that has been applied consistently at a spatial resolution of $30 \mathrm{~m}$ across the U.S.A.. NLCD is based primarily on the unsupervised classification of Landsat satellite data. NLCD 2001 and 2006 are geo-registered to the Albers Equal Area projection grid [47]. There are 16 land-cover classes in NLCD. Introducing too many classes would affect the spectral unmixing accuracy for linear mixture model applied to multispectral image. The original 16 land-cover classes were combined and 
reclassified into 4 classes, namely, Water-Wetlands (WW), Developed-Barren (DB), Shrubland-Herbaceous (SH), and Planted/Cultivated (PC).

The reference fine-resolution map was generated from NLCD 2001, and the input fine-resolution map was generated from NLCD 2006. Both maps contain $800 \times$ 800 pixels located in Charlotte (Fig. 3). The changed fine-resolution pixels account for $7.8 \%$ of all pixels in the study area. The input coarse-resolution multispectral image was simulated from the fine-resolution reference map. A fine-resolution multispectral image was produced first. The number of bands was set to 6 to accommodate for a dimensionality constraint in spectral unmixing. The 4 endmember signature digital number $(\mathrm{DN})$ values were set to $[750,400,225,20,80,130]^{\mathrm{T}},[310$, $70,107,390,360,330]^{\mathrm{T}},[160,295,455,605,720,960]^{\mathrm{T}}$, and $[440,520,750,890$, $980,520]^{\mathrm{T}}$. T is the transposition operator. The covariance matrices for all the classes were set to $\mathrm{A} \cdot \mathbf{I}$, where $\mathrm{A}=500$ is a constant and $\mathbf{I}$ is 6 by 6 identity matrix [42]. The fine-resolution multispectral image was first produced according to the reference map and class endmembers. The spectral response of each class was normally distributed in each waveband. The fine-resolution multispectral image was then spatially degraded to the coarse-resolution multispectral image with the scale factor $s=10$. The reference fine-resolution land-cover change/no-change map and from-to change map were produced by a per-pixel comparison of the NLCD 2001 and 2006 land-cover maps (Fig. 3). 

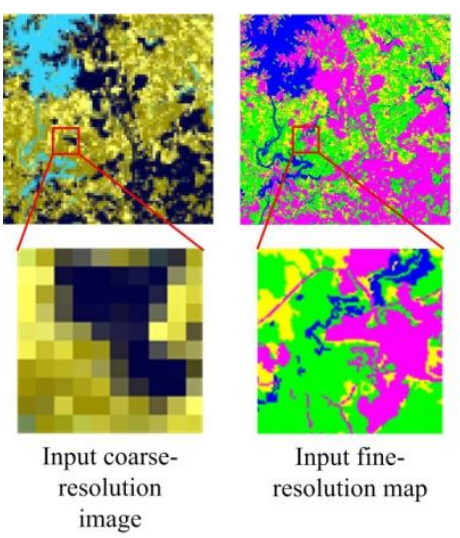

Input fine-

resolution map

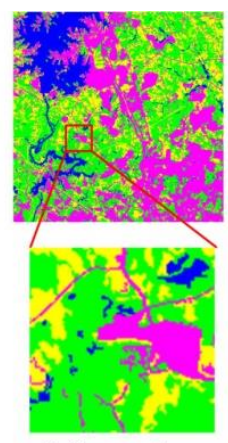

Reference fineresolution map

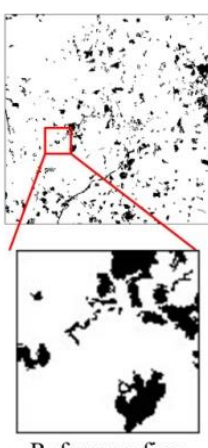

Reference fineresolution change/no-change map

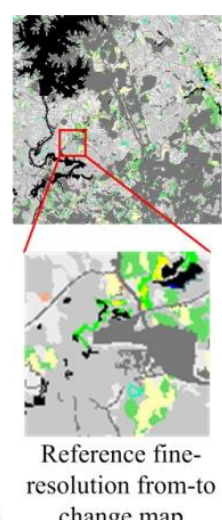

change map
Land-cover map legend

WW

$\square$ DB

$\square \mathrm{SH}$

PC

From-to change map legend

Change/no-change map legend

Changed

Unchanged

Unchanged WW

Unchanged DB

Unchanged SH

Unchanged PC

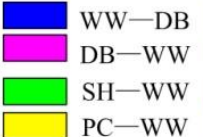

WW- $\mathrm{SH}$

$\mathrm{DB}-\mathrm{SH}$

$\mathrm{SH}-\mathrm{DB}$

$\mathrm{PC}-\mathrm{DB}$

Fig. 3. SRCD input and reference images of synthetic image experiment. The zoomed area contains $100 \times 100$ fine-resolution pixels.

The initial SRCD parameters were set. The initial class area proportion change threshold value, $t_{i n i}$, can be set between 0 and 1 . A high $t_{i n i}$ value would extend the SRCD convergence time, whereas a low $t_{i n i}$ value would eliminate small-sized unchanged land-cover patches in the intermediate change/no-change map. Thus, the initial class area proportion change threshold value was set to $t_{i n i}=0.5$. A low $\Delta t$ value would extend the SRCD convergence time, whereas a high $\Delta t$ value would reduce the number of unchanged land-cover patches in the intermediate and final change/no-change maps. Thus, the step class area proportion change threshold value was set to $\Delta t=-0.05$. The number of total iterations was set to ite $e_{\text {total }}=20 . \mathrm{SRCD}$ is terminated with a class area proportion change threshold value of $t=t_{\text {ini }}+$ ite $_{\text {total }} \times \Delta t=-0.5$. The SRCD results are shown in Fig. 4 .

\section{2) Results}




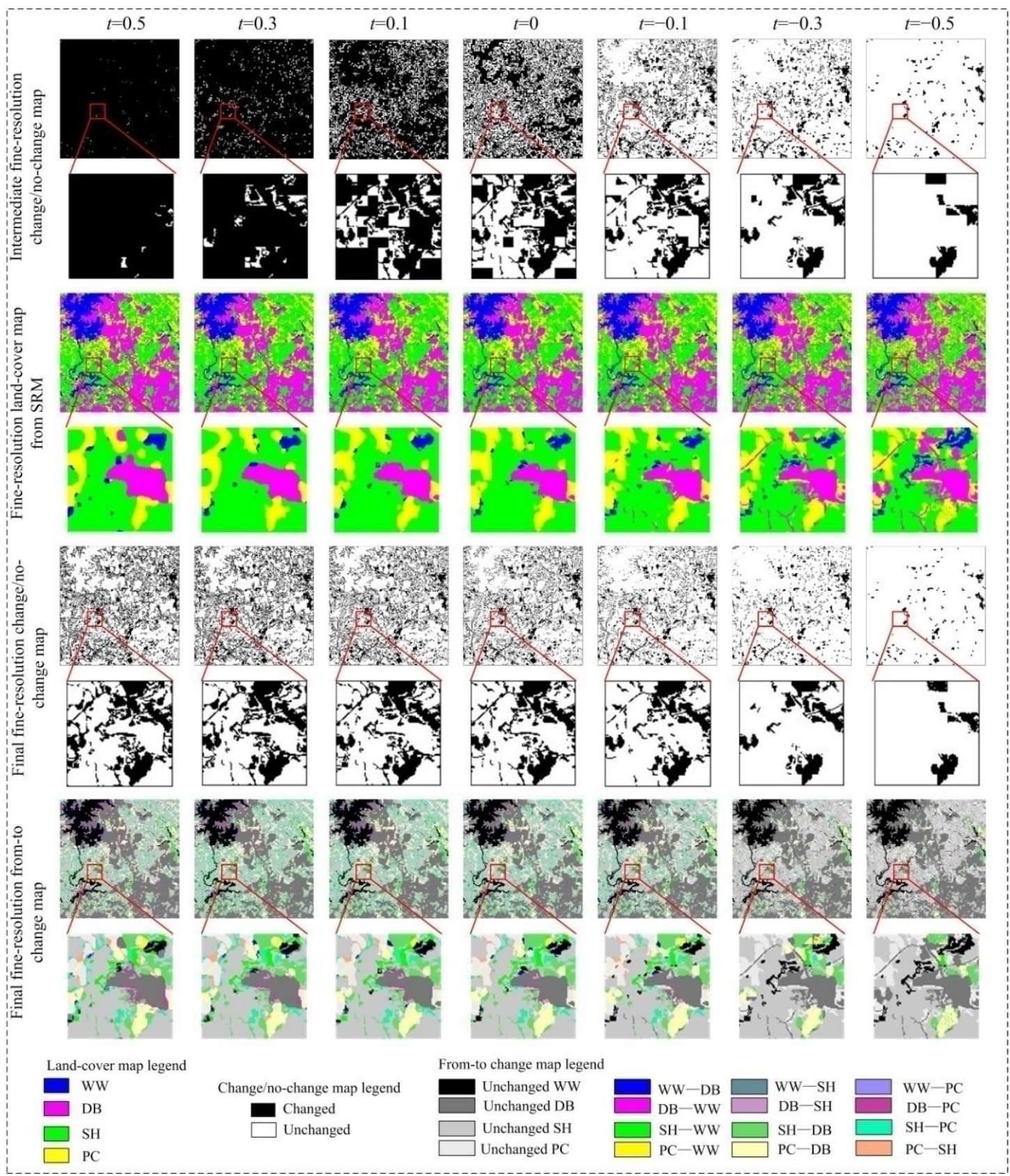

Fig. 4. SRCD result maps of synthetic image experiment at different class area proportion change threshold $t$ for synthetic image experiment. The zoomed area contains $100 \times 100$ fine-resolution pixels.

Fig. 4 summarizes the SRCD results, highlighting the intermediate fine-resolution change/no-change maps used for SRM, the fine-resolution land-cover maps generated by SRM, and the final output fine-resolution change/no-change maps and from-to change maps at different class area proportion change threshold $t$ values. More fine-resolution pixels were indicated as having changed labels in the intermediate change/no-change map than in the final output change/no-change maps at different threshold $t$ values. This result may be attributed to the application of SRM 
to the fine-resolution pixels indicated as changed in the intermediate change/no-change map, which indicated parts of the changed pixels in the intermediate change/no-change map as unchanged pixels in the final change/no-change map.

The performance of SRCD varied with the class area proportion change threshold $t$. When the threshold is $t=0.3$, most fine-resolution pixels were indicated as having changed in the intermediate change/no-change map. The changed fine-resolution pixel labels were determined by SRM. Many rounded patches were evident in the subset images because SRM adopted the land-cover maximum spatial dependence model, which could have oversmoothed the land-cover patches. With the decrease in threshold $t$, more fine-resolution pixels were detected as unchanged in the intermediate change/no-change map, and more land-cover patches in the input fine-resolution map, which were identified as unchanged, were preserved in the land-cover map produced by SRM. More unchanged fine-resolution pixels were also observed in the final fine-resolution change/no-change and from-to change maps. When the threshold is $t=-0.3$, most fine-resolution pixel labels in the input fine-resolution map with unchanged class area proportion were preserved in the land-cover map, and the final change/no-change and from-to change maps were approximate to the reference maps.

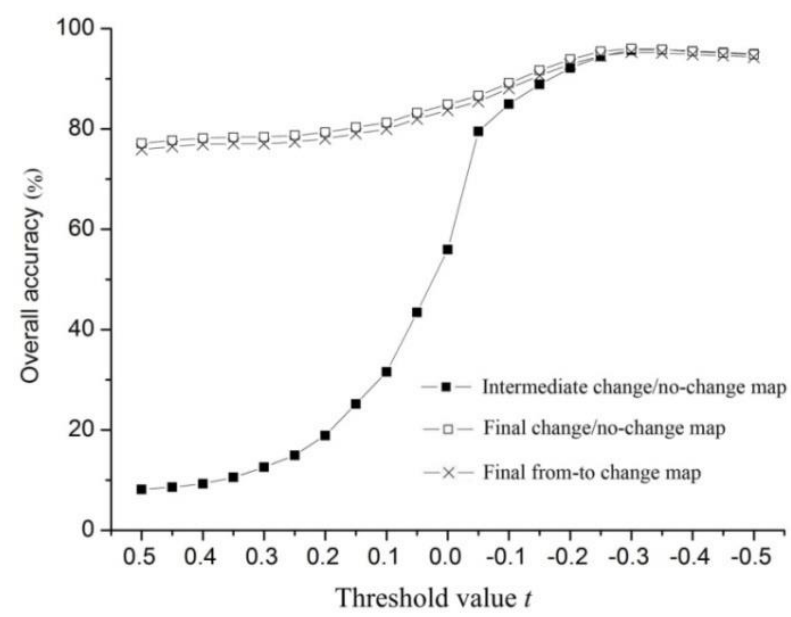

Fig. 5. Overall accuracies of intermediate change/no-change maps, final change/no-change maps, and final from-to change maps for synthetic image.

The overall accuracies of the intermediate and final change/no-change maps, as 
well as the final from-to change maps, are shown in Fig. 5. The overall accuracies of all the maps increased gradually with a decrease in threshold $t$ when $t$ is higher than -0.3 and decreased with a decrease in threshold $t$ when $t$ is lower than -0.3 . The final change/no-change maps exhibited a higher overall accuracy than the intermediate change/no-change maps for each threshold $t$ value. This result is due to difference in the data sources used for generating these maps: the intermediate change/no-change map was produced using the class area proportion information at the coarse-resolution scale, whereas the final change/no-change map was produced by comparing land-cover maps at the fine-resolution scale. The highest overall accuracies of the final change/no-change map and the from-to change maps were $96.00 \%$ and $95.28 \%$, respectively, when $t=-0.3$.
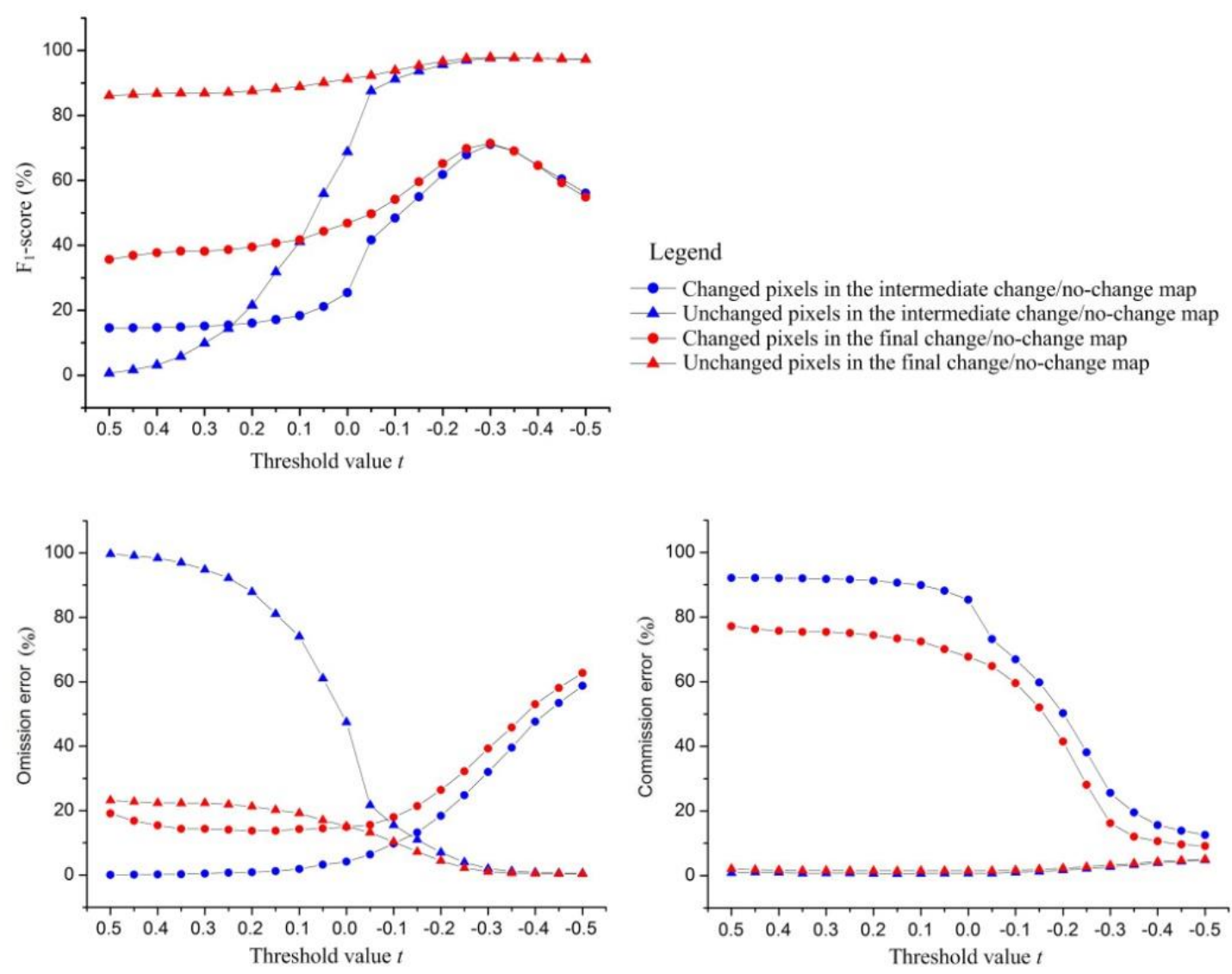

Fig. 6. The $\mathrm{F}_{1}$-scores, omission errors, and commission errors of unchanged and changed fine-resolution pixels in the intermediate and final fine-resolution change/no-change maps for the synthetic image experiment.

The accuracies of the intermediate and final change/no-change maps were measured using the $\mathrm{F}_{1}$-score, omission error and commission error, which are shown 
in Fig. 6. The $F_{1}$-score for the changed pixels in the intermediate and final change/no-change maps increased gradually with a decrease in threshold $t$ when $t$ is higher than -0.3 , and decreased when the threshold $t$ is lower than -0.3 because many changed pixels were erroneously identified as unchanged pixels. The intermediate and final change/no-change maps have the highest $\mathrm{F}_{1}$-score (higher than $71 \%$ ) for changed pixels when the threshold $t=-0.3$. The $\mathrm{F}_{1}$-scores for unchanged pixels in the intermediate and final change/no-change maps increased gradually with a decrease in threshold $t$ when $t$ is higher than -0.3 and remained almost unchanged when the threshold $t$ is lower than -0.3 . The highest $\mathrm{F}_{1}$-score for unchanged pixels was higher than that for changed pixels in the intermediate and final change/no-change maps. This is because most pixels were unchanged in the study area, and the majority of pixels were identified as unchanged by SRCD when the threshold $t=-0.3$. The final change/no-change maps produced by comparing land-cover maps at the fine-resolution scale have higher $F_{1}$-scores for changed and unchanged pixels than the intermediate change/no-change maps produced using the class area proportion information at the coarse-resolution scale at most threshold $t$ values.

The omission errors of unchanged pixels decreased with a decrease in threshold $t$. This result is because most unchanged fine-resolution pixels were initially indicated as having changed; however, with a decrease in threshold $t$, the detected unchanged fine-resolution pixels increased, and more unchanged fine-resolution pixels were correctly detected. By contrast, the omission errors of changed pixels increased with a decrease in threshold $t$ because almost all fine-resolution pixels were initially detected as having changed. With a decrease in threshold $t$, the detected changed fine-resolution pixels decreased, and more actually changed pixels were erroneously identified as unchanged.

The commission errors of changed pixels decreased with a decrease in threshold $t$. This result is because almost all the fine-resolution pixels were initially detected as having changed; however, with a decrease in threshold $t$, the detected changed fine-resolution pixels decreased, and many of the initially erroneously detected changed pixels were excluded from the detected changed pixels. The commission 
errors of unchanged pixels were low and remained almost unchanged with a decrease in threshold $t$. When threshold $t$ is lower than -0.3 , the commission errors of the unchanged pixels increased, whereas the commission errors of the changed pixels decreased with a decrease in threshold $t$. Therefore, more changed pixels were erroneously detected as unchanged, and less unchanged pixels were erroneously detected as changed.

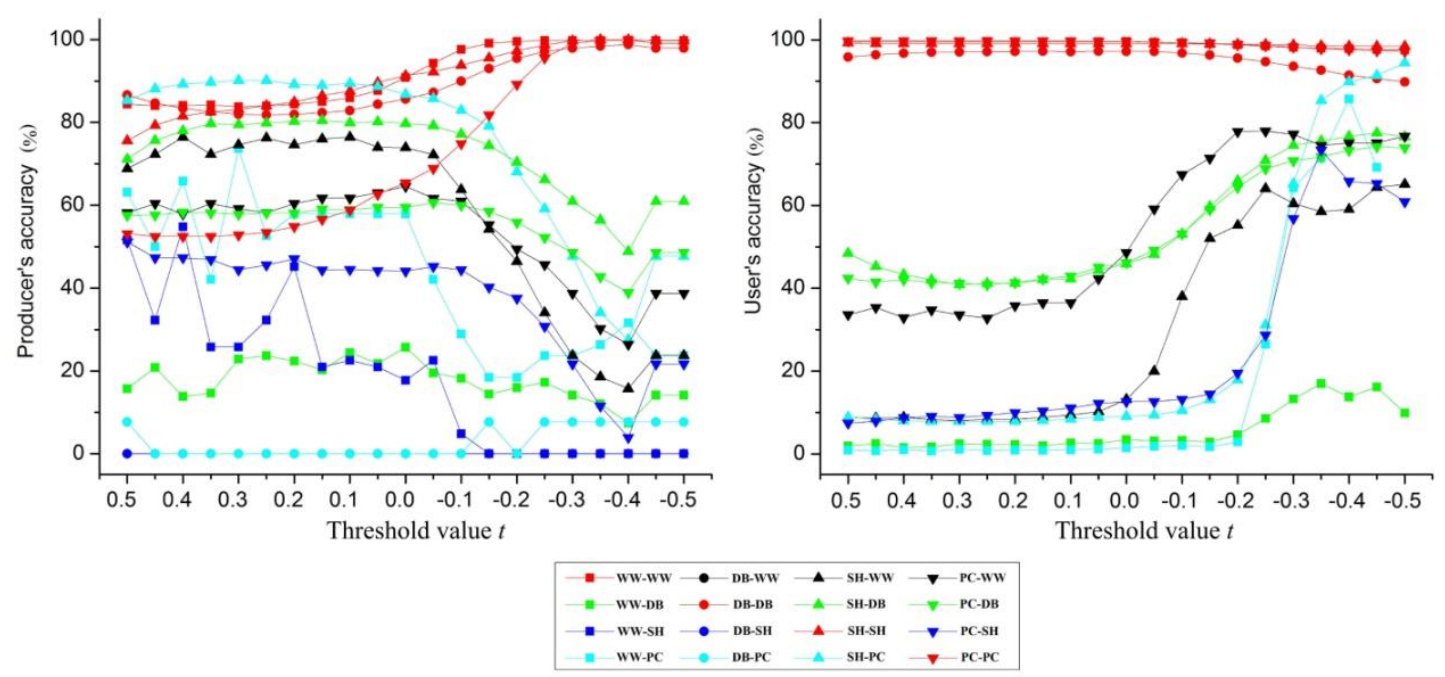

Fig. 7. The producer's accuracy and user's accuracy of different land-cover from-to changes for synthetic image experiment.

The producer's accuracy and user's accuracy of different land-cover from-to changes for the synthetic image experiment are shown in Fig. 7. The producer's accuracies of $\mathrm{WW}-\mathrm{WW}, \mathrm{DB}-\mathrm{DB}, \mathrm{SH}-\mathrm{SH}$, and $\mathrm{PC}-\mathrm{PC}$ increased with a decrease in threshold $t$, whereas the producer's accuracies of the other land-cover changes decreased with a decrease in threshold $t$. The number of correctly detected unchanged classes increased, whereas the number of correctly detected changed classes decreased. The producer's accuracies of $\mathrm{DB}-\mathrm{PC}$ and $\mathrm{WW}-\mathrm{SH}$ were $0 \%$ because only 13 fine-resolution pixels had a DB-PC change and 62 fine-resolution pixels had a WW-SH change in the 2001-2006 reference from-to change map. These changed patches were smaller than a coarse-resolution pixel, which contained $s^{2}=100$ fine-resolution pixels. The producer's accuracies of unchanged classes have a dominant effect on the global accuracy in the from-to change map because more than $90 \%$ of the fine-resolution pixels were unchanged in 2001-2006. In Fig. 7, the user's 
accuracies of unchanged classes decreased, whereas those of the changed classes increased with a decrease in threshold $t$. Fig. 8 shows the reference endmember signatures, extracted initial endmember signatures at $t=0.5$, and extracted final endmember signatures at $t=-0.3$. A good match between the reference and estimated endmember signatures was observed at $t=-0.3$.

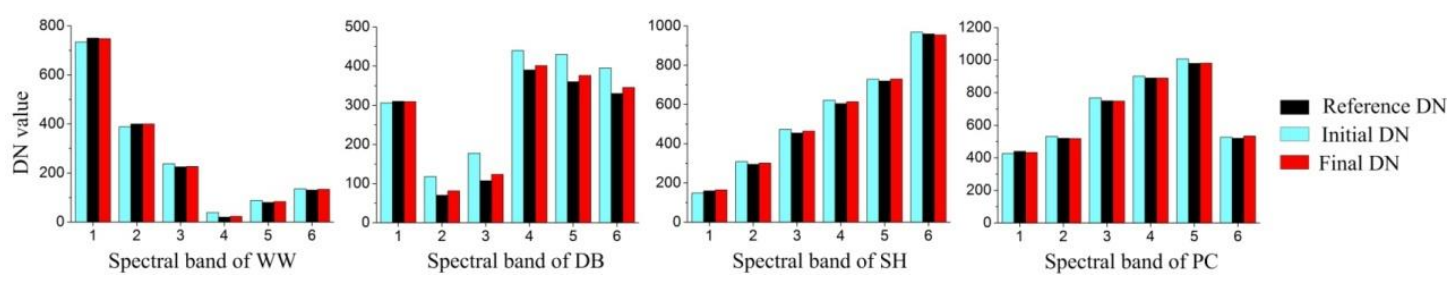

Fig. 8. Reference endmember signatures (reference DN), estimated endmember signatures at $t=0.5$ (initial DN) and at $t=-0.3$ (final DN) for the synthetic image experiment.

\section{B. MODIS Image Experiment}

\section{1) Data Preparation}

MODIS multispectral images were adopted to assess SRCD based on real remotely sensed images. The study area is located near Sorriso (12 $33^{\prime} 21$ "S and $55^{\circ} 42^{\prime} 31^{\prime \prime}$ ) in Mato Grosso State, Brazil. This area is in the Brazilian Amazon Basin, and is mainly covered by tropical forests but which has undergone a deforestation in recent years. The experiment data include a Landsat-5 TM image with a spatial resolution of $30 \mathrm{~m}$ acquired on August 13, 2000, a Landsat-7 ETM+ image with a spatial resolution of $30 \mathrm{~m}$ acquired on July 18, 2005, and a single eight-day surface reflectance MODIS product (MOD09A1) with a spatial resolution of $463 \mathrm{~m}$ taken in July 2005. The TM image was geo-registered to the ETM+ image. The TM image acquired in 2000 was digitized to the input fine-resolution land-cover map with forest and nonforest in it, and the ETM+ image acquired in 2005 was digitized to the reference map. The MODIS image comprising 7 spectral bands (620 nm - $2055 \mathrm{~nm}$ ) was used as the SRCD input coarse-resolution image. The MODIS image was re-projected into the Universal Transverse Mercator coordinate system and then resampled to a spatial resolution of $450 \mathrm{~m}$ using the nearest neighbor algorithm. A subset of $200 \times 200$ pixels MODIS image and subsets of $3000 \times 3000$ pixels TM and 
ETM+ images were adopted (Fig. 9). The scale factor $s$ is set to 15 . The changed fine-resolution pixels account for $22.8 \%$ of all pixels in the study area.

SRCD was implemented for the scenarios in which the fine-resolution map pre-dated the coarse-resolution image. The reference fine-resolution land-cover change/no-change map and from-to change map were produced by a per-pixel comparison of the 2000 and 2005 land-cover maps. The SRCD parameters were set as in the synthetic image experiment: $t_{i n i}=0.5, \Delta t=-0.05$, and ite $_{\text {total }}=20$.

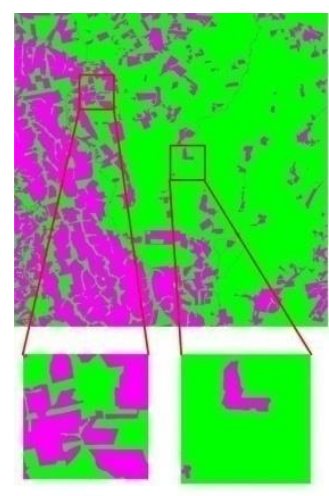

2000 fine-resolution map

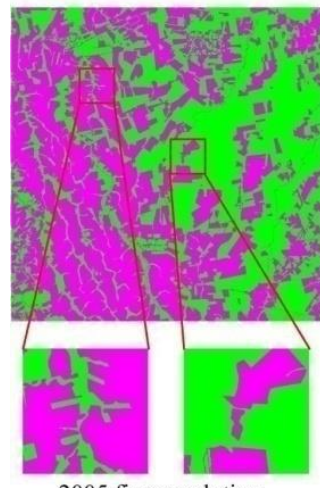

2005 fine-resolution reference map

Land-cover map legend

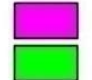

Nonforest

Forest

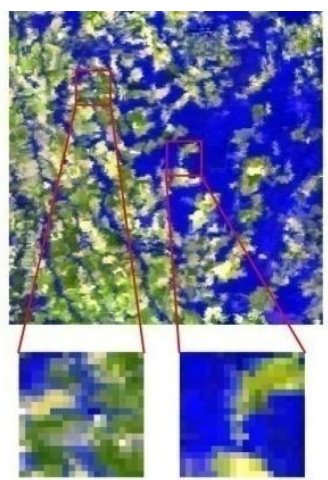

2005 MODIS coarse-resolutiuon input image

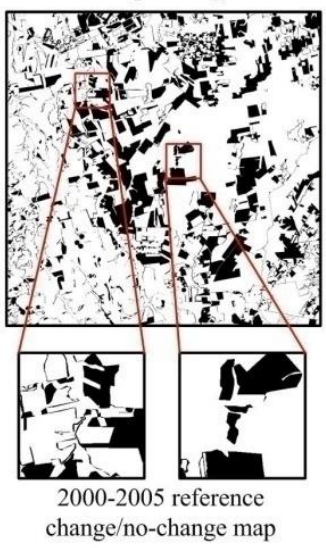

Change/no-change map legend

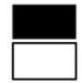

Changed

Unchanged
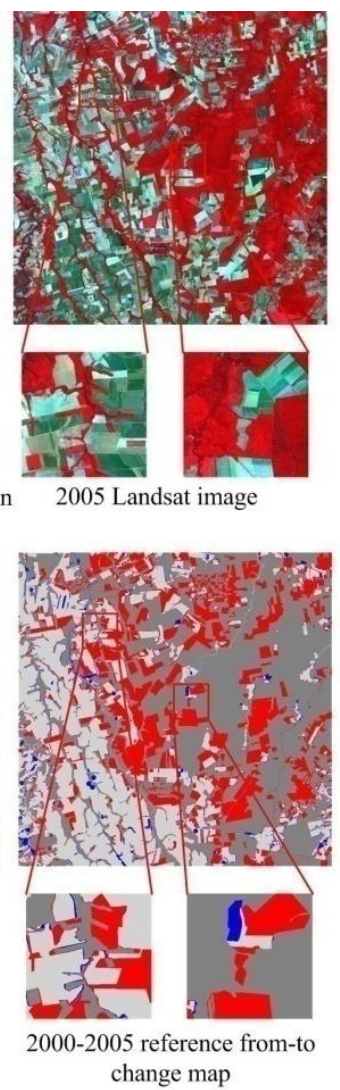

From-to change map legend

Unchanged forest Unchanged nonforest Forest-nonforest Nonforest-forest

Fig. 9. SRCD input and reference images of MODIS image experiment. The zoomed area contains $300 \times 300$ fine-resolution pixels.

\section{2) Results}




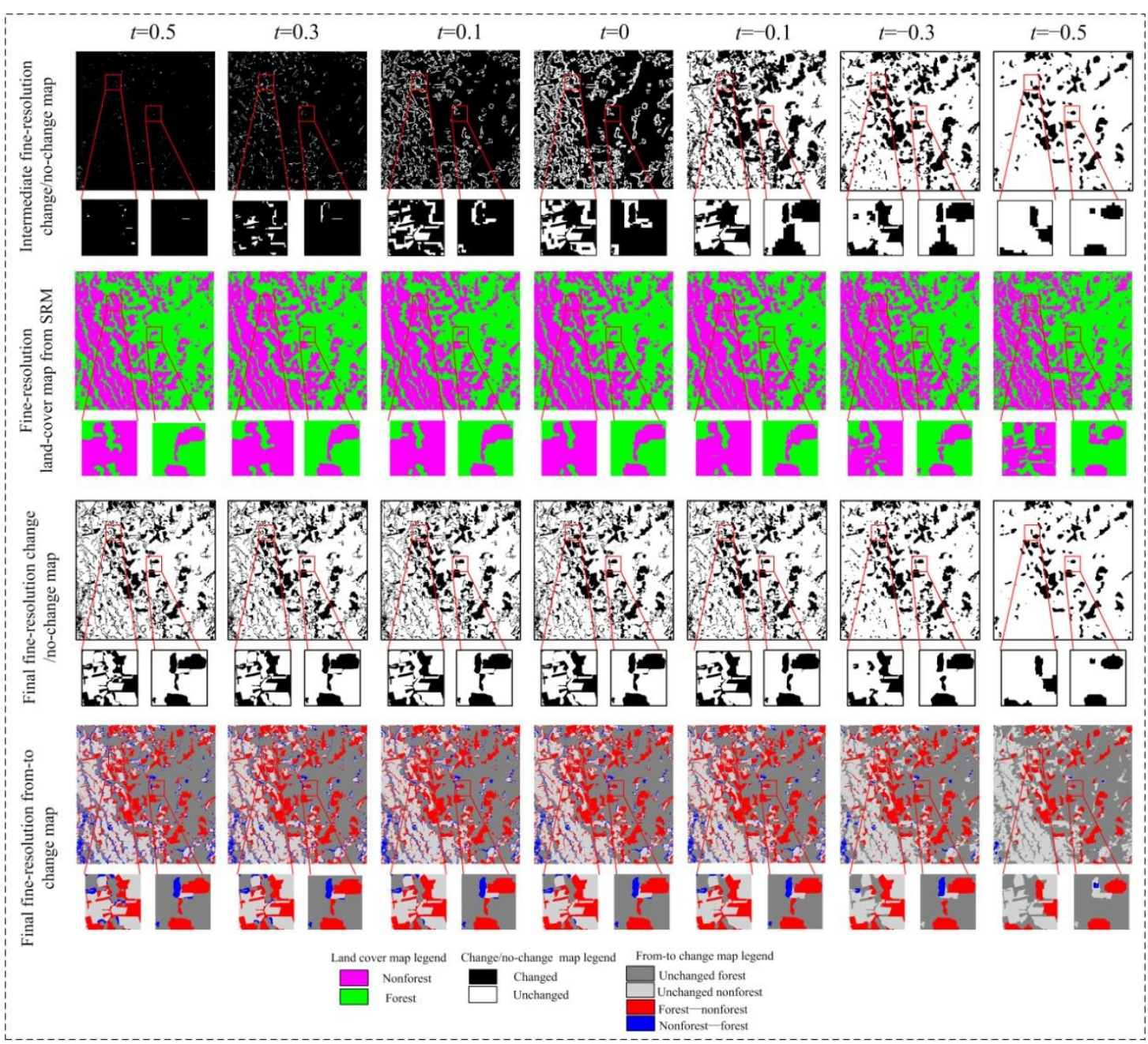

Fig. 10. Experimental results of MODIS image experiment

The input images, reference maps, and result maps are shown in Fig. 9 and Fig. 10. Deforestation occurred in the region during the periods represented. Some of the deforestations exhibited a fishbone pattern because of the expansion of the nonforest patch (such as the left zoomed area), whereas some of the deforestations were in clear-cut areas (such as the right zoomed area). More pixels were detected as unchanged pixels with a decrease in threshold $t$. As a result, the changed pixels marked in black decreased, whereas the unchanged pixels marked in white increased in the intermediate and final change/no-change maps. The changed land-cover trajectories marked in blue and red decreased, whereas the unchanged land-cover trajectories marked in gray increased in the from-to change maps with a decrease in threshold $t$. In the reference from-to change map in Fig. 9, forest-nonforest change trajectory patches marked in red were more than nonforest-forest change trajectory patches marked in blue because deforestation was the main land-cover change 
trajectory in the study area. Most of the spatial patterns of the changed land-cover trajectories were correctly mapped onto the from-to change map when $t=-0.3$. The forest-nonforest patches were mapped with rounded shape, and some of the spatial details were lost. This result is attributed to the use of the land-cover maximal spatial dependence model by SRM in producing the changed land-cover patches; this model could oversmooth class boundaries.

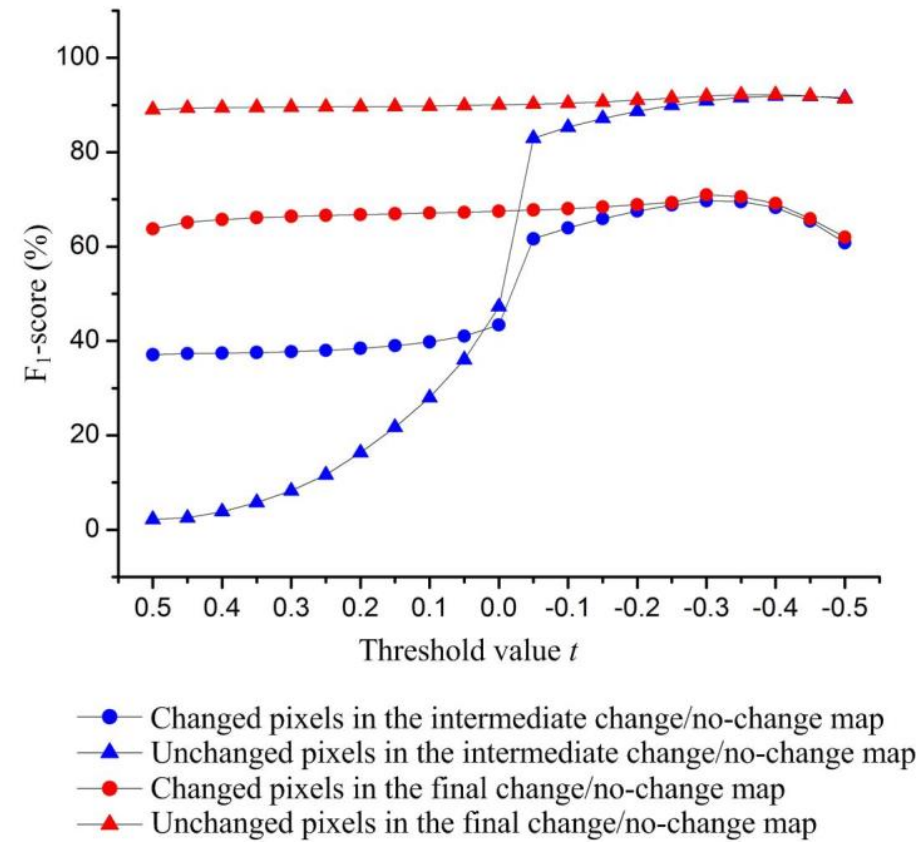

Fig. 11. The $\mathrm{F}_{1}$-scores for changed and unchanged pixels in the intermediate and final fine-resolution change/no-change maps for MODIS image experiment.

Fig. 11 shows the $\mathrm{F}_{1}$-scores for changed and unchanged pixels in the intermediate and final fine-resolution change/no-change maps. For changed pixels, the intermediate change/no-change maps obtained $\mathrm{F}_{1}$-scores lower than $50 \%$ when $t>0$ and obtained $\mathrm{F}_{1}$-scores higher than $60 \%$ when $t<0$. For unchanged pixels, the $\mathrm{F}_{1}$-scores obtained by the intermediate change/no-change maps were lower than $50 \%$ when $t>0$ and higher than $80 \%$ when $t<0$. The final change/no-change maps have $\mathrm{F}_{1}$-scores higher than $60 \%$ for changed pixels and higher than $90 \%$ for unchanged pixels. The highest $\mathrm{F}_{1}$-score of the final change/no-change map is approximately $70.97 \%$ for changed pixels when $t=-0.3$ and approximately $92.18 \%$ for unchanged pixels when $t=-0.4$. 


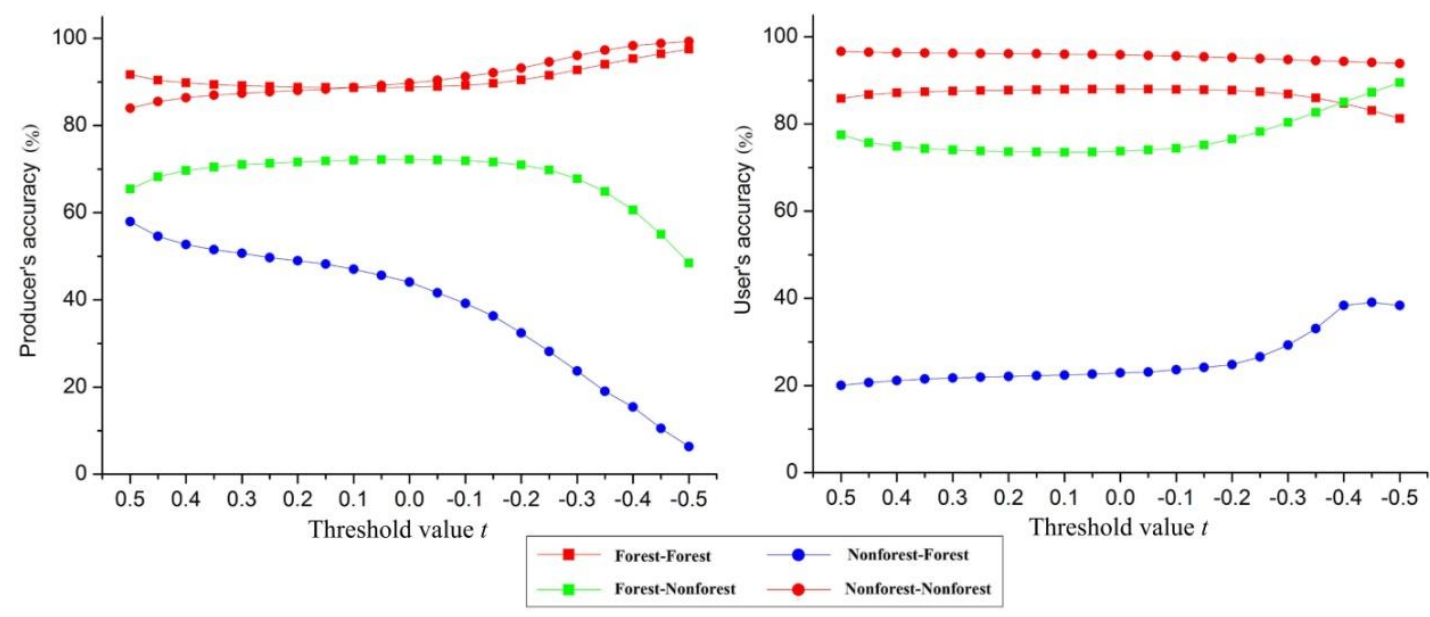

Fig. 12. The producer's accuracy and user's accuracy of different land-cover from-to changes for MODIS experiment.

Fig. 12 shows the producer's and user's accuracies of different land-cover from-to changes at different threshold $t$ values. The producer's accuracies of nonforest-nonforest and forest-forest changes increased, whereas the producer's accuracies of forest-nonforest and nonforest-forest changes decreased because more fine-resolution pixels were detected as unchanged with a decrease in threshold $t$. The producer's accuracies of nonforest-nonforest and forest-forest changes were higher than those for forest-nonforest and nonforest-forest changes. This result is because the unchanged pixel labels were determined by the input fine-resolution land-cover map, whereas the changed pixel labels were determined by SRM. The producer's accuracy of forest-nonforest change was higher than that for nonforest-forest change. The main reason is that the forest-nonforest patches were usually larger than the coarse-resolution pixel; thus, SRM with the maximum land-cover spatial dependence model was suitable for mapping these patches [24]. By contrast, nonforest-forest patches were usually found along patch boundaries with linear shapes wherein the SRM may have oversmoothed the patches. The pixel number of the nonforest-forest change accounts for $2.0 \%$ of all the pixels, and the pixel number of the forest-nonforest change accounts for $20.7 \%$ of all the pixels in the 2000-2005 reference fine-resolution from-to change map. Thus, the forest-nonfores change detection accuracy has a more dominant effect on the overall change detection accuracy of the entire image. The user's accuracies of unchanged classes decreased, 
whereas those of changed classes increased with a decrease in threshold $t$. This result is because the number of pixels of changed classes marked in blue and red decreased, whereas the number of pixels of unchanged classes marked in gray increased with a decrease in threshold $t$. The overall accuracy of the form-to change map increased from $83.27 \%$ when $t=0.5$ to $87.15 \%$ when $t=-0.3$ and then decreased to $85.98 \%$ when $t=-0.5$.

\section{Landsat-8 OLI Image Experiment}

\section{1) Data Preparation}

A Landsat multispectral images was adopted as the coarse-resolution image in this experiment. The study area is located in Wuhan $\left(30^{\circ} 21^{\prime} 70^{\prime \prime} \mathrm{N}\right.$ and $\left.114^{\circ} 15^{\prime} 19^{\prime \prime} \mathrm{E}\right)$, China. A Landsat-8OLI image acquired on June 13, 2013 was selected as the relative coarse-resolution image. The Landsat-8 OLI image has 9 multispectral bands. The first 7 bands of OLI image with a spatial resolution of $30 \mathrm{~m}$ were selected as the coarse-resolution image for SRCD input. The $8^{\text {th }}$ band (panchromatic band) of OLI image was not used because it has a spatial resolution of $15 \mathrm{~m}$, and the $9^{\text {th }}$ band (cirrus band) of OLI image was not used because it is not suitable for detecting land-covers. Two Google Earth optical images with fine spatial resolution, which were acquired on August 14, 2010 and on June 13, 2013 were selected. The Google Earth optical images were geo-registered to the ETM+ image. The 2010 Google Earth image was digitized to the SRCD input previous fine-resolution land-cover map, and the 2013 Google Earth image was digitized to the reference map. There are 4 land-cover classes in the fine-resolution maps, which are water, grass, impervious surface and bareland. A subset of $40 \times 40$ pixels Landsat- 8 OLI image was used as the SRCD input coarse-resolution image. The previous and reference land-cover maps contains $200 \times 200$ pixels (Fig. 13). The scale factor $s$ is set to 5 . The changed fine-resolution pixels account for $12.5 \%$ of all pixels in the study area. The reference fine-resolution land-cover change/no-change map and from-to change map were produced by a per-pixel comparison of the 2010 and 2013 land-cover maps. The SRCD parameters 
were set as in the synthetic and MODIS image experiments: $t_{i n i}=0.5, \Delta t=-0.05$, and ite $_{\text {total }}=20$.

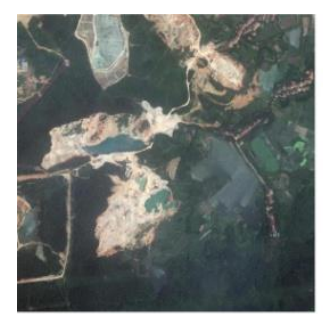

2010 fine-resolution

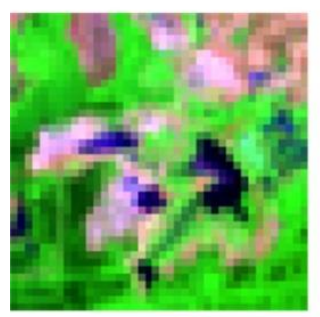

2013 coarse-resolution (bands: 7(SWIR2)-5(NIR)-3(Green))

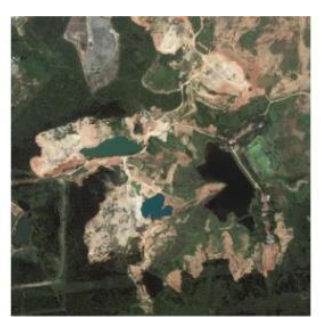

2013 fine-resolution

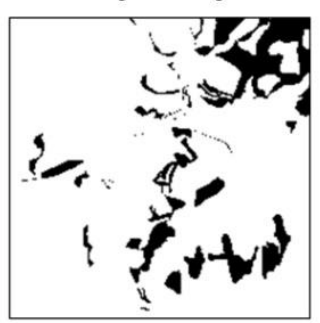

2010-2013 reference
change/no-change map

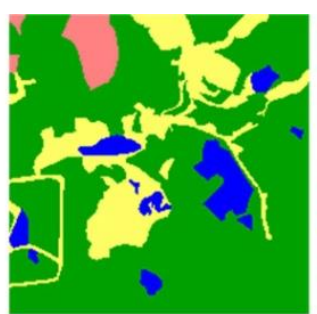

2010 fine-resolution

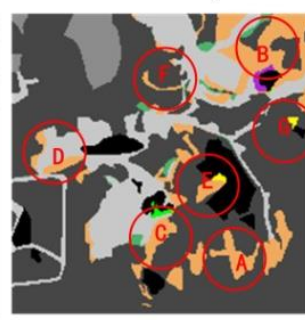

2010-2013 reference

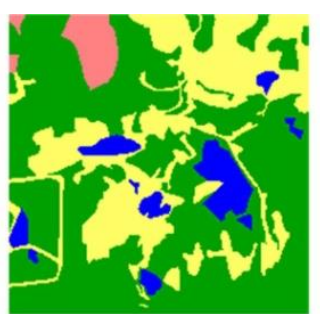

2013 finc-resolution

Land-cover map (reference map)

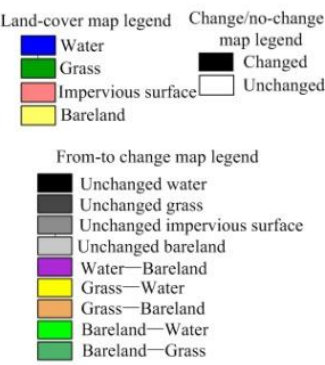

Fig. 13. SRCD input and reference images of Landsat-8 OLI image experiment.

2) Results
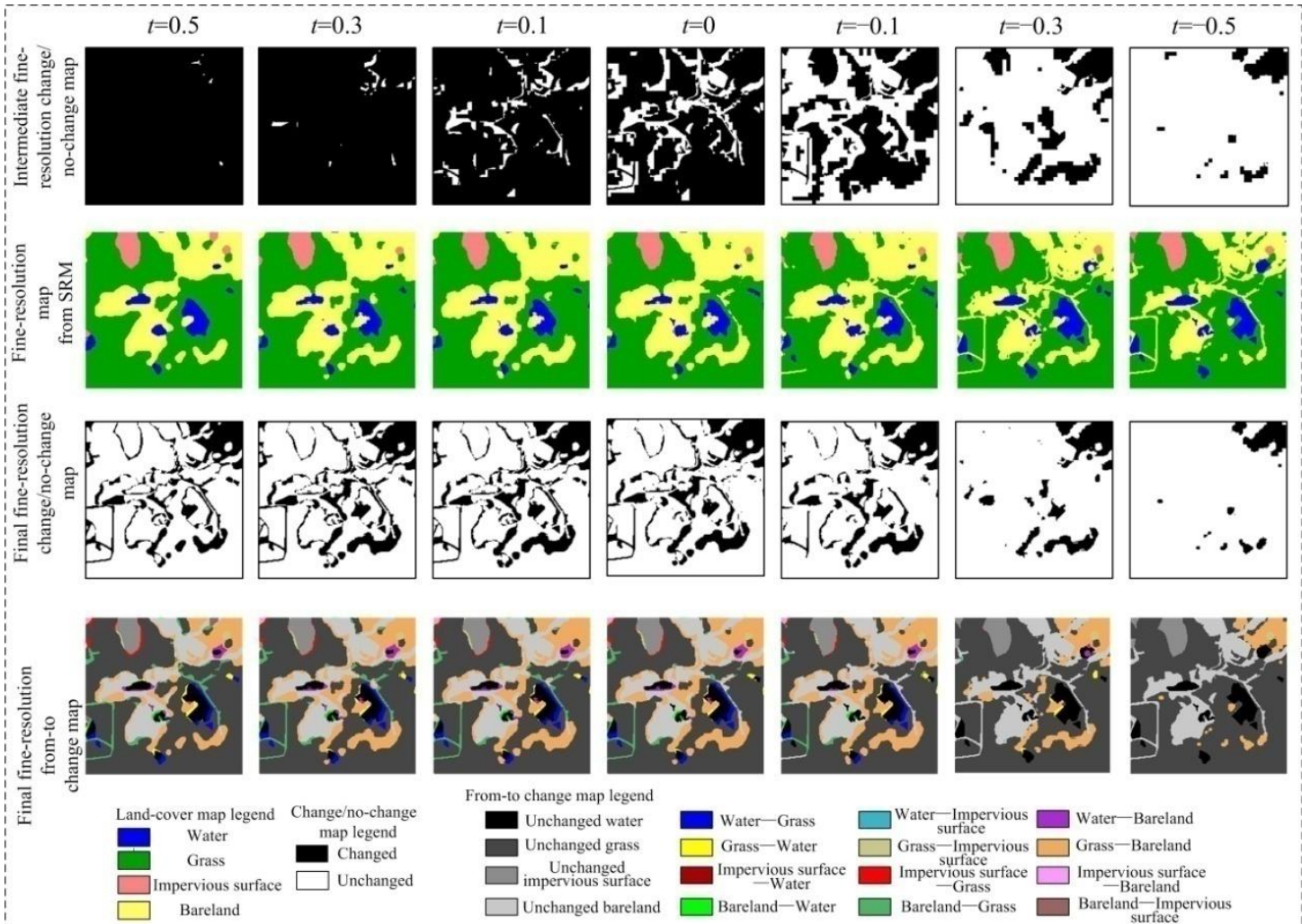

From-to change map legend

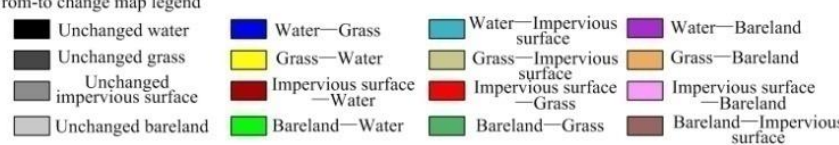

Fig. 14. Experimental results of Landsat-8 OLI image experiment.

As shown in Fig. 13, an obvious grass-bareland change was found in the image 
in subsets $\mathrm{A}$ to $\mathrm{F}$ in the reference from-to change map. The grass-bareland changes in subsets $\mathrm{C}, \mathrm{D}$, and $\mathrm{E}$ were mainly caused by the expansion of the previously bareland. Other land-cover changes were unremarkable, such as in subset G. In Fig. 13, more pixels were identified as unchanged pixels with a decrease in threshold $t$. In the final change/no-change maps, the changed pixels marked in black decreased, whereas the unchanged pixels marked in white increased with a decrease in threshold $t$. In the final from-to change maps, the land-cover changes marked in color decreased with a decrease in threshold $t$. The erroneously detected from-to changes (bareland-grass and water-grass) in the bottom-left corner of the image appeared when $t>-0.3$ and disappeared when $t \leq-0.3$. This result may be attributed to the fact that the bareland and water patches in the bottom-left corner were small in the reference map and were detected as changed and oversmoothed by SRM when $t>-0.3$. The grass-bareland changes in subsets A to E were detected in the from-to change maps when $t>-0.5$. The shapes of the changed patches were smoothed because of the spatial smoothing effect of SRM. As a result, the grass-bareland changes in subsets A and B had rounded shapes; being smaller than the coarse-resolution pixel, grass-bareland changes in subset $\mathrm{F}$ were eliminated in the from-to change map because of the oversmoothing effect of SRM. The grass-bareland changes in subset C were detected as having a linear shape when $t>-0.5$ and were similar to those in the reference from-to change map in Fig. 13. Some of the changed patches that were smaller than the coarse-resolution pixel of other from-to changes were detected when $t>-0.5$ (such as grass-water changes in subset G). 


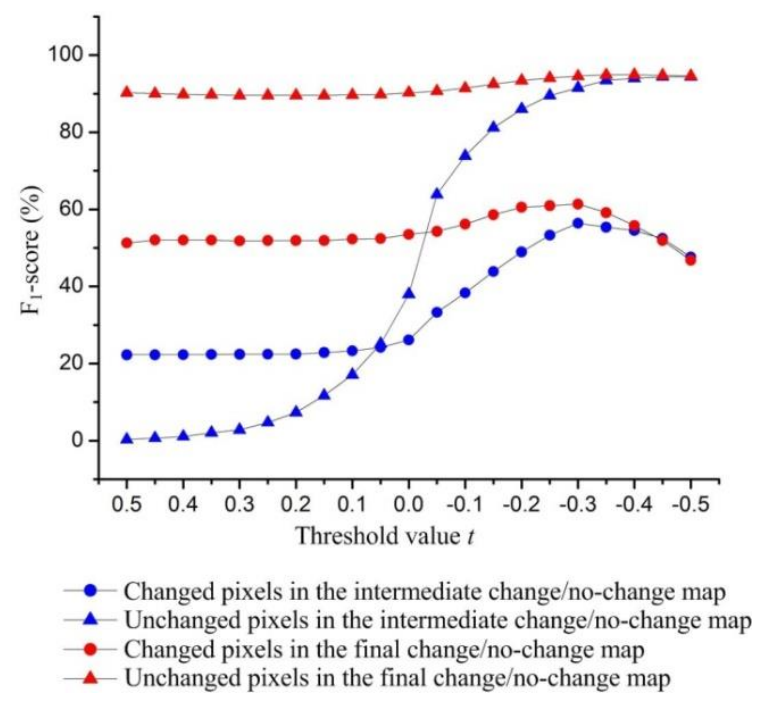

Fig. 15. The $\mathrm{F}_{1}$-scores for changed and unchanged pixels in the intermediate and final fine-resolution change/no-change maps for Landsat image experiment.

Fig. 15 shows the $F_{1}$-scores for changed and unchanged pixels in the intermediate and final fine-resolution change/no-change maps. The $\mathrm{F}_{1}$-scores for changed pixels in the intermediate and final change/no-change maps increased gradually with a decrease in threshold $t$ when $t>-0.3$, and decreased when the $t<-0.3$. The $\mathrm{F}_{1}$-score for unchanged pixels in the intermediate and final change/no-change maps gradually increased with a decrease in threshold $t$ when $t>-0.3$ and remained almost unchanged when $t<-0.3$. The highest $\mathrm{F}_{1}$-score for changed pixels was $56.39 \%$ in the intermediate change/no-change map when $t=-0.3$ and $61.37 \%$ in the final change/no-change map when $t=-0.3$.

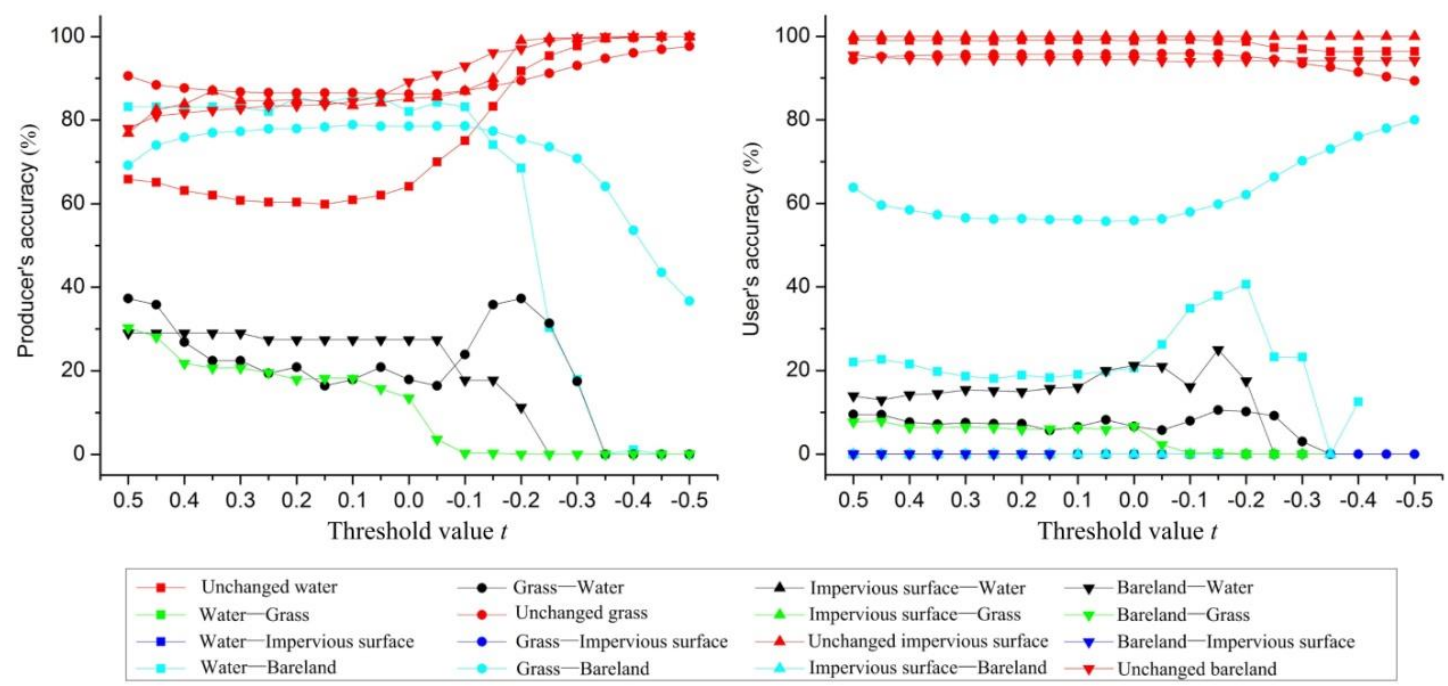

Fig. 16. The producer's accuracies and user's accuracies of different land-cover from-to changes for the Landsat-8 OLI image experiment. 
Fig. 16 shows the producer's and user's accuracies of different land-cover from-to changes at different values of threshold $t$. The producer's accuracies of unchanged land-covers increased, whereas the producer's accuracies of changed land-covers decreased with a decrease in threshold $t$. The producer's accuracy of grass-bareland change was higher than $70 \%$ when $t>-0.3$, and the producer's accuracies of grass-water, bareland-water, and bareland-grass changes were lower than $40 \%$. As shown in Table II, the grass-bareland change accounts for $11.06 \%$ of all the fine-resolution pixels in the image. By contrast, the water-bareland, grass-water, bareland-water, and bareland-grass changes account for $0.22 \%, 0.17 \%, 0.15 \%$, and $0.91 \%$ of all the fine-resolution pixels, respectively, in the reference from-to change map. The majority of fine-resolution pixels of water-bareland change and of grass-bareland change were correctly detected in the final from-to change maps in Table II. As shown in Fig. 13, the land-cover patches of water-bareland, grass-water, bareland--water, and bareland-grass changes were small. The fine-resolution pixels of water-bareland, grass-water, and bareland-water changes account for less than 4 coarse-resolution pixels (100 fine-resolution pixels) in the reference from-to change map, as shown in Table II. As a result, the class area proportion changes of the from-to changes were insignificant and can hardly be detected. In addition, these changed patches are likely to be oversmoothed by the spatial smoothing effect of SRCD. By contrast, the land-cover patches of grass-bareland changes were larger than the coarse-resolution pixel and were not oversmoothed by SRCD. The overall accuracy of the from-to change map increased from $83.37 \%$ when $t=0.5$ to $85.42 \%$ when $t=-0.1$ and to $90.15 \%$ when $t=-0.3$. 
TABLE II

THE NUMBER OF PIXELS IN THE REFERENCE AND RESULT MAPS OF DIFFERENT LAND-COVER FROM-TO CHANGES FOR THE LANDSAT-8 OLI IMAGE EXPERIMENT.

\begin{tabular}{lccccc}
\hline $\begin{array}{l}\text { Land-cover } \\
\text { from-to change } \\
\text { type }\end{array}$ & $\begin{array}{l}\text { Number of pixels } \\
\text { in the reference } \\
\text { from-to change } \\
\text { map }\end{array}$ & $\begin{array}{l}\text { Percentage of } \\
\text { pixels in the } \\
\text { reference from-to } \\
\text { change map }\end{array}$ & \multicolumn{2}{l}{$\begin{array}{l}\text { Number of correctly detected pixels } \\
\text { in the result from-to change map }\end{array}$} \\
\cline { 5 - 7 } water-bareland & 89 & $0.22 \%$ & 74 & 61 & 16 \\
grass-water & 67 & $0.17 \%$ & 16 & 25 & 5 \\
grass-bareland & 4423 & $11.06 \%$ & 3475 & 3334 & 3131 \\
bareland-water & 62 & $0.15 \%$ & 11 & 7 & 0 \\
bareland-grass & 363 & $0.91 \%$ & 1 & 0 & 0 \\
\hline
\end{tabular}

\section{CONCLUSIONS}

Land-cover changes are usually detected using bi-temporal fine-resolution images. Given that fine-spatial-resolution images have low temporal resolutions, the land-cover changes are detected infrequently. Compared with fine-resolution images, coarse-resolution images have high temporal repetition rates. Considering the trade-off between spatial and temporal resolutions, land-cover changes can be detected using both fine- and coarse-resolution images. In this paper, an SRCD method is proposed. It uses a coarse-resolution image and a fine-resolution map to detect land-cover changes more temporally, continuously, and frequently and provide fine spatial resolution. In SRCD, the endmember signatures and fine-resolution land-cover maps are iteratively estimated and updated. The unchanged fine-resolution pixels are iteratively determined using an intermediate change/no-change map while the changed fine-resolution pixel labels are iteratively updated using SRM. The method generates the following outputs: a fine-resolution change/no-change map and a fine-resolution from-to change map.

The performance of SRCD was validated using synthetic, MODIS, and Landsat-8 OLI multispectral images of scenarios in which the fine-resolution map pre-dated and post-dated the coarse-resolution image. In the three experiments, the accuracies of the output fine-resolution change/no-change and from-to change maps 
generated using SRCD varied with the threshold value. In the change/no-change maps, the omission errors of the unchanged pixels decreased, whereas the omission errors of the changed pixels increased with a decrease in threshold. In the change/no-change maps, the $\mathrm{F}_{1}$-score for changed pixels increased with a decrease in threshold when the threshold is higher than -0.3 and decreased when the threshold is lower than -0.3 . In the from-to change maps, the land-cover from-to changes, which account for the majority of the land-cover change pixels, were correctly detected, and the changed land-cover patches that were larger than the coarse-resolution pixel were correctly mapped. In all experiments, the highest change detection accuracies were observed at a threshold of approximately -0.3 . The land-cover change/no-change maps have the highest $\mathrm{F}_{1}$-score for changed pixels, reaching higher than $70 \%$ in the synthetic image and MODIS image experiments and higher than 55\% in the Landsat image experiment. In all three experiments, the from-to change maps achieved the highest overall accuracy of more than $85 \%$.

In the three experiments, some of the changed land-cover patches that were smaller than the coarse-resolution pixel were not detected and mapped onto the from-to change maps. Several factors may reduce the detection accuracy for small-sized land-cover patches. First, a linear mixture model was adopted to estimate the endmembers with the use of the coarse-resolution image and fine-resolution map and estimate the class area proportions with the use of the coarse-resolution image and the estimated endmembers. However, endmember variances caused by spatial changes were not handled. Furthermore, violating the linearity assumption of the mixture models, the multiple scattering of photons between different surface components causes errors in the SRCD endmembers and class area proportion estimations. Powerful spectral unmixing algorithms, such as the multiple endmember spectral mixture analysis and nonlinear mixture model, should be developed in SRCD. Second, SRCD adopted the maximal land-cover spatial dependence model to characterize the spatial distribution of land-cover patches that were detected to have changed in SRCD. This spatial dependence model is suitable for characterizing the shapes of objects that are larger than the coarse-resolution pixel. However, doing so 
may oversmooth objects that are smaller than the coarse-resolution pixel. The integration with other spatial dependence models, such as geostatistical models that are more suitable for characterizing objects that are smaller than the coarse-resolution pixel, should be studied in SRCD. Finally, the performance of SRCD is related to the spectral and spatial resolutions of the coarse-resolution remotely sensed image. In general, when the coarse-resolution input has a higher spectral resolution, the interclass separabilities are high; as a result, both endmember estimation accuracy and spectral unmixing accuracy are expected to be high. When the coarse-resolution input has a finer spatial resolution, fewer land-cover classes are contained in the coarse-resolution pixel and the mixed pixel effect is less severe, thus the class area proportions could be estimated more accurately. Accordingly, the intermediate land-cover change/no-change map can be generated more accurately based on the estimated class area proportions from the coarse-resolution image. The performance of SRCD, which updates the changed fine-resolution pixels in the intermediate change/no-change map, can thus be improved when the coarse-resolution image has high spectral and spatial resolutions.

\section{Acknowledgement:}

This work was supported in part by Natural Science Foundation of Hubei Province for Distinguished Young Scholars under Grant No. 2013CFA031, in part by the National Basic Research Program (973 Program) of China under Grant 2013cb733205, in part by the Natural Science Foundation of China under Grant No. 41301398, and in part by the Wuhan ChenGuang Youth Sci.\&Tech. Project under Grant 2014072704011254.

\section{References:}

[1] S. E. Franklin, and M. A. Wulder, "Remote sensing methods in medium spatial resolution satellite data land cover classification of large areas," Progress in Physical Geography, vol. 26, no. 2, pp. 173-205, Jun, 2002.

[2] B. L. Turner, W. B. Meyer, and D. L. Skole, "Global land-use/land-cover change: towards an integrated study," AMBIO, vol. 23, no. 1, pp. 91-95, Feb, 1994.

[3] T. L. Sohl, "Change analysis in the United Arab Emirates: An investigation of techniques," 
Photogramm. Eng. Remote Sens., vol. 65, no. 4, pp. 475-484, Apr, 1999.

[4] J. R. G. Townshend, and C. O. Justice, "Spatial variability of images and the monitoring of changes in the normalized difference vegetation index," Int. J. Remote Sens., vol. 16, no. 12, pp. 2187-2195, Aug, 1995.

[5] E. F. Lambin, and A. H. Strahler, "Change-vector analysis in multitemporal space: a tool to detect and categorize land-cover change processes using high temporal-resolution satellite data," Remote Sens. Environ., vol. 48, no. 2, pp. 231-244, May, 1994.

[6] D. Lu, P. Mausel, E. Brondizio et al., "Change detection techniques," Int. J. Remote Sens., vol. 25, no. 12, pp. 2365-2407, Jun, 2004.

[7] J. B. Adams, D. E. Sabol, V. Kapos et al., "Classification of multispectral images based on fractions of endmembers: application to land-cover change in the Brazilian Amazon," Remote Sens. Environ., vol. 52, no. 2, pp. 137-154, May, 1995.

[8] A. Erturk, and A. Plaza, "Informative change detection by unmixing for hyperspectral images," IEEE Geosci. Remote Sens. Lett., vol. 12, no. 6, pp. 1252-1256, Jun, 2015.

[9] D. Lu, M. Batistella, E. Moran et al., "Fractional forest cover mapping in the Brazilian Amazon with a combination of MODIS and TM images," Int. J. Remote Sens., vol. 32, no. 22, pp. 7131-7149, 2011, 2011.

[10] D. S. Lu, M. Batistella, and E. Moran, "Multitemporal spectral mixture analysis for Amazonian land-cover change detection," Can. J. Remote Sens., vol. 30, no. 1, pp. 87-100, Feb, 2004.

[11] S. Le Hegarat-Mascle, C. Ottle, and C. Guerin, "Land cover change detection at coarse spatial scales based on iterative estimation and previous state information," Remote Sens. Environ., vol. 95, no. 4, pp. 464-479, Apr, 2005.

[12] P. M. Atkinson, "Sub-pixel target mapping from soft-classified, remotely sensed imagery," Photogramm. Eng. Remote Sens., vol. 71, no. 7, pp. 839-846, Jul, 2005.

[13] F. Ling, Y. Du, X. D. Li et al., "Superresolution land cover mapping with multiscale information by fusing local smoothness prior and downscaled coarse fractions," IEEE Trans. Geosci. Remote Sensing, vol. 52, no. 9, pp. 5677-5692, Sep, 2014.

[14] A. J. Tatem, H. G. Lewis, P. M. Atkinson et al., "Super-resolution target identification from remotely sensed images using a Hopfield neural network," IEEE Trans. Geosci. Remote Sensing, vol. 39, no. 4, pp. 781-796, Apr, 2001.

[15] T. Kasetkasem, M. K. Arora, and P. K. Varshney, "Super-resolution land cover mapping using a Markov random field based approach,” Remote Sens. Environ., vol. 96, no. 3-4, pp. 302-314, Jun 30, 2005.

[16] X. D. Li, Y. Du, and F. Ling, "Spatially adaptive smoothing parameter selection for Markov random field based sub-pixel mapping of remotely sensed images,” Int. J. Remote Sens., vol. 33, no. 24, pp. 7886-7901, 2012.

[17] X. D. Li, F. Ling, Y. Du et al., "Spatially adaptive superresolution land cover mapping with multispectral and panchromatic images," IEEE Trans. Geosci. Remote Sensing, vol. 52, no. 5, pp. 2810-2823, May, 2014.

[18] F. Ling, Y. Du, F. Xiao et al., "Subpixel land cover mapping by integrating spectral and spatial information of remotely sensed imagery," IEEE Geosci. Remote Sens. Lett., vol. 9, no. 3, pp. 408-412, May, 2012.

[19] F. Ling, Y. Du, X. D. Li et al., "Interpolation-based super-resolution land cover mapping,” Remote Sens. Lett., vol. 4, no. 7, pp. 629-638, Jul 1, 2013. 
[20] Q. M. Wang, W. Z. Shi, and L. G. Wang, "Allocating classes for soft-then-hard subpixel mapping algorithms in units of class," IEEE Trans. Geosci. Remote Sensing, vol. 52, no. 5, pp. 2940-2959, May, 2014.

[21] Y. Ge, S. P. Li, and V. C. Lakhan, "Development and testing of a subpixel mapping algorithm," IEEE Trans. Geosci. Remote Sensing, vol. 47, no. 7, pp. 2155-2164, Jul, 2009.

[22] Y. H. Zhang, Y. Du, F. Ling et al., "Example-based super-resolution land cover mapping using support vector regression," IEEE J. Sel. Top. Appl. Earth Observ. Remote Sens., vol. 7, no. 4, pp. 1271-1283, Apr, 2014.

[23] X. Xu, Y. F. Zhong, and L. P. Zhang, "Adaptive subpixel mapping based on a multiagent system for remote-sensing imagery," IEEE Trans. Geosci. Remote Sensing, vol. 52, no. 2, pp. 787-804, Feb, 2014.

[24] P. M. Atkinson, "Issues of uncertainty in super-resolution mapping and their implications for the design of an inter-comparison study," Int. J. Remote Sens., vol. 30, no. 20, pp. 5293-5308, 2009.

[25] F. Ling, W. Li, Y. Du et al., "Land cover change mapping at the subpixel scale with different spatial-resolution remotely sensed imagery," IEEE Geosci. Remote Sens. Lett., vol. 8, no. 1, pp. 182-186, Jan, 2011.

[26] X. D. Li, Y. Du, and F. Ling, "Super-resolution mapping of forests with bitemporal different spatial resolution images based on the spatial-temporal Markov random field," IEEE J. Sel. Top. Appl. Earth Observ. Remote Sens., vol. 7, no. 1, pp. 29-39, Jan, 2014.

[27] Y. Xu, and B. Huang, "A spatio-temporal pixel-swapping algorithm for subpixel land cover mapping," IEEE Geosci. Remote Sens. Lett., vol. 11, no. 2, pp. 474-478 2014.

[28] X. D. Li, F. Ling, Y. Du et al., "A spatial-temporal Hopfield neural network approach for super-resolution land cover mapping with multi-temporal different resolution remotely sensed images," ISPRS J. Photogramm. Remote Sens., vol. 93, pp. 76-87, Jul, 2014.

[29] A. Bateson, and B. Curtiss, "A method for manual endmember selection and spectral unmixing," Remote Sens. Environ., vol. 55, no. 3, pp. 229-243, Mar, 1996.

[30]J. Boardman, F. Kruse, and R. Green, "Mapping target signatures via partial unmixing of AVIRIS," Proc. Summaries JPL Airborne Earth Sci. Workshop, pp. 23-26, 1995.

[31] M. E. Winter, "N-FINDR: an algorithm for fast autonomous spectral end-member determination in hyperspectral data," Imaging Spectrometry $V$, Proceedings of the Society of Photo-Optical Instrumentation Engineers (Spie) M. R. Descour and S. S. Shen, eds., pp. 266-275, 1999.

[32] A. Plaza, P. Martinez, R. Perez et al., "Spatial/spectral endmember extraction by multidimensional morphological operations," IEEE Trans. Geosci. Remote Sensing, vol. 40, no. 9, pp. 2025-2041, Sep, 2002.

[33] H. Li, and L. Zhang, "A hybrid automatic endmember extraction algorithm based on a local window," IEEE Trans. Geosci. Remote Sensing, vol. 49, no. 11, pp. 4223-4238, Nov, 2011.

[34] A. Plaza, P. Martinez, R. Perez et al., "A quantitative and comparative analysis of endmember extraction algorithms from hyperspectral data," IEEE Trans. Geosci. Remote Sens., vol. 42, no. 3, pp. 650-663, Mar, 2004.

[35] G. M. Foody, "Supervised image classification by MLP and RBF neural networks with and without an exhaustively defined set of classes," Int. J. Remote Sens., vol. 25, no. 15, pp. 3091-3104, Aug, 2004.

[36] G. M. Foody, "Hard and soft classifications by a neural network with a non-exhaustively defined set of classes," Int. J. Remote Sens., vol. 23, no. 18, pp. 3853-3864, Sep, 2002. 
[37] R. Zurita-Milla, J. Clevers, and M. E. Schdepman, "Unmixing-based Landsat TM and MERIS FR data fusion," IEEE Geosci. Remote Sens. Lett., vol. 5, no. 3, pp. 453-457, Jul, 2008.

[38] J. Settle, and N. Campbell, "On the errors of two estimators of sub-pixel fractional cover when mixing is linear," IEEE Trans. Geosci. Remote Sens., vol. 36, no. 1, pp. 163-170, Jan, 1998.

[39] J. J. Settle, and N. A. Drake, "Linear mixing and the estimation of ground cover proportions," Int. J. Remote Sens., vol. 14, no. 6, pp. 1159-1177, Apr, 1993.

[40] Y. E. Shimabukuro, and J. A. Smith, "The least-squares mixing models to generate fraction images derived from remote sensing multispectral data," IEEE Trans. Geosci. Remote Sens., vol. 29, no. 1, pp. 16-20, Jan, 1991.

[41] W. R. Tobler, "A computer movie simulating urban growth in the Detroit region," Economic Geography, vol. 46, pp. 234-240, 1970.

[42] V. A. Tolpekin, and A. Stein, "Quantification of the effects of land-cover-class spectral separability on the accuracy of Markov-random-field-based superresolution mapping," IEEE Trans. on Geosci. and Remote Sens., vol. 47, no. 9, pp. 3283-3297, 2009.

[43] H. Aghighi, J. Trinder, S. Lim et al., "Fully spatially adaptive smoothing parameter estimation for Markov random field super-resolution mapping of remotely sensed images," Int. J. Remote Sens., vol. 36, no. 11, pp. 2851-2879, 2015.

[44] S. Geman, and D. Geman, "Stochastic relaxation, Gibbs distributions, and the Bayesian restoration of the images," IEEE Trans. Pattern Anal. Mach. Intell., vol. 6, no. 6, pp. 721-741, 1984.

[45] M. K. Ridd, and J. J. Liu, "A comparison of four algorithms for change detection in an urban environment," Remote Sens. Environ., vol. 63, no. 2, pp. 95-100, Feb, 1998.

[46] C. J. v. Rijsbergen, Information Retrieval, 2nd ed. : Woburn, MA: Butterworth-Heinemann, 1979.

[47] C. Homer, C. Q. Huang, L. M. Yang et al., "Development of a 2001 national land-cover database for the United States," Photogramm. Eng. Remote Sens., vol. 70, no. 7, pp. 829-840, Jul, 2004. 